ACCEPTED FOR PUBLICATION IN THE ASTROPHYSICAL JOURNAL

Preprint typeset using $\mathrm{LT}_{\mathrm{E}} \mathrm{X}$ style emulateapj v. 05/04/06

\title{
THE DEARTH OF UV-BRIGHT STARS IN M32: IMPLICATIONS FOR STELLAR EVOLUTION THEORY ${ }^{1}$
}

\author{
Space Telescope Science Institute, 3700 San Martin Drive, Baltimore, MD 21218; tbrown@stsci.edu, edsmith@stsci.edu, ferguson@stsci.edu
}

\author{
Allen V. Sweigart, Randy A. Kimble, Charles W. Bowers \\ Code 667, NASA Goddard Space Flight Center, Greenbelt, MD 20771; allen.v.sweigart@nasa.gov, randy.a.kimble@nasa.gov, charles.w.bowers@nasa.gov \\ Accepted for publication in The Astrophysical Journal
}

\begin{abstract}
Using the Space Telescope Imaging Spectrograph on the Hubble Space Telescope, we have obtained deep farultraviolet images of the compact elliptical galaxy M32. When combined with earlier near-ultraviolet images of the same field, these data enable the construction of an ultraviolet color-magnitude diagram of the hot horizontal branch (HB) population and other hot stars in late phases of stellar evolution. We find few post-asymptotic giant branch (PAGB) stars in the galaxy, implying that these stars either cross the HR diagram more rapidly than expected, and/or that they spend a significant fraction of their time enshrouded in circumstellar material. The predicted luminosity gap between the hot HB and its AGB-Manqué (AGBM) progeny is less pronounced than expected, especially when compared to evolutionary tracks with enhanced helium abundances, implying that the presence of hot $\mathrm{HB}$ stars in this metal-rich population is not due to $\Delta Y / \Delta Z \gtrsim 4$. Only a small fraction $(\sim 2 \%)$ of the HB population is hot enough to produce significant UV emission, yet most of the UV emission in this galaxy comes from the hot HB and AGBM stars, implying that PAGB stars are not a significant source of UV emission even in those elliptical galaxies with a weak UV excess.

Subject headings: galaxies: evolution - galaxies: stellar content - galaxies: individual (M32) - stars: evolution - stars: horizontal branch
\end{abstract}

\section{INTRODUCTION}

The nearest elliptical galaxy, M32 (NGC221), provides a useful testing ground for stellar evolution theory. With solarblind ultraviolet (UV) observations that suppress the dominant cool population, hot stars in late evolutionary phases can be resolved into the center of the galaxy. From such observations one can construct a UV color-magnitude diagram (CMD) for a large sample of hot stars so that these rapid evolutionary phases can be studied in detail and compared with theoretical predictions. In contrast, the rapid evolution of these stars means few of them are found in Galactic globular clusters (the traditional testing ground for stellar evolution), while distance and reddening uncertainties hamper their study in the Galactic field population.

In an elliptical galaxy with little or no star formation, the UV-bright stars will consist of stars residing on the hot end of the horizontal branch (HB) itself, also known as the extreme HB (EHB), as well as stars that have evolved beyond the HB phase. Although the HB morphology tends to be red at high metallicity ( $Z$; the "first parameter"), EHB stars are found in the Galactic field, metal-rich elliptical galaxies, some of the more massive metal-rich globular clusters, and metal-rich open clusters. The existence of EHB stars in old metal-rich populations demonstrates that parameters besides metallicity play a role in HB morphology, driving the "second parameter" debate, and producing much controversy regarding the source of the UV emission (also known as the UV upturn or UV excess) in quiescent elliptical galaxies. Both resolved imaging (Brown et al. 2000b) and integrated spectroscopy (Brown et al. 1997; Ferguson et al. 1991) imply that EHB stars and their progeny are the source of this emission, but it is unclear what parameters affect the large variation in the UV-to-optical

\footnotetext{
${ }^{1}$ Based on observations made with the NASA/ESA Hubble Space Telescope, obtained at STScI, and associated with proposal 9053.
}

flux from galaxy to galaxy (O'Connell 1999; Yi et al. 1999; Tantalo et al. 1996; Dorman et al. 1995; Park \& Lee 1997; Greggio \& Renzini 1990). Because a star's effective temperature on the HB is a function of its mass, possible candidates for these parameters include age and helium abundance, since both strongly affect the mass at which a star leaves the main sequence.

For a population with a given age and metallicity, the stars will arrive on the HB with nearly the same helium core mass $\left(\sim 0.5 M_{\odot}\right)$ but a range of hydrogen envelope mass $(\sim 0.001-$ $0.3 M_{\odot}$ ), determined by the range in mass lost during the ascent up the red giant branch (RGB). This range in the envelope mass produces a range in effective temperature, with the coolest stars having the largest envelope mass. After $\sim 100$ Myr, helium is depleted in the convective core, and the star leaves the HB. Its subsequent evolution to the white dwarf (WD) cooling curve will occur along one of three possible paths (Figure 1), also depending upon the envelope mass. Greggio \& Renzini (1990) give a complete review of these paths, which we briefly summarize here.

The reddest $\mathrm{HB}$ stars (with the most massive envelopes) ascend the asymptotic giant branch (AGB). Near the bright end of the AGB, they undergo thermal pulses, alternating between longer periods of quiescent hydrogen shell burning and shorter periods of energetic helium shell flashes. During the AGB phase, the hydrogen envelope is reduced both by mass loss (e.g., stellar winds) from the surface and by hydrogen shell burning at the base of the envelope. Once the envelope mass is reduced below a critical value $\left(M_{c r i t}\right)$, a star will leave the AGB and rapidly cross the HR diagram as a bright postAGB (PAGB) star, possibly forming a planetary nebula along the way, before descending the WD cooling curve. If the star leaves the AGB between thermal pulses, it crosses as an $\mathrm{H}$ burning PAGB star, but if it leaves during a thermal pulse, it crosses as an He-burning PAGB star. Due to their rapid evo- 


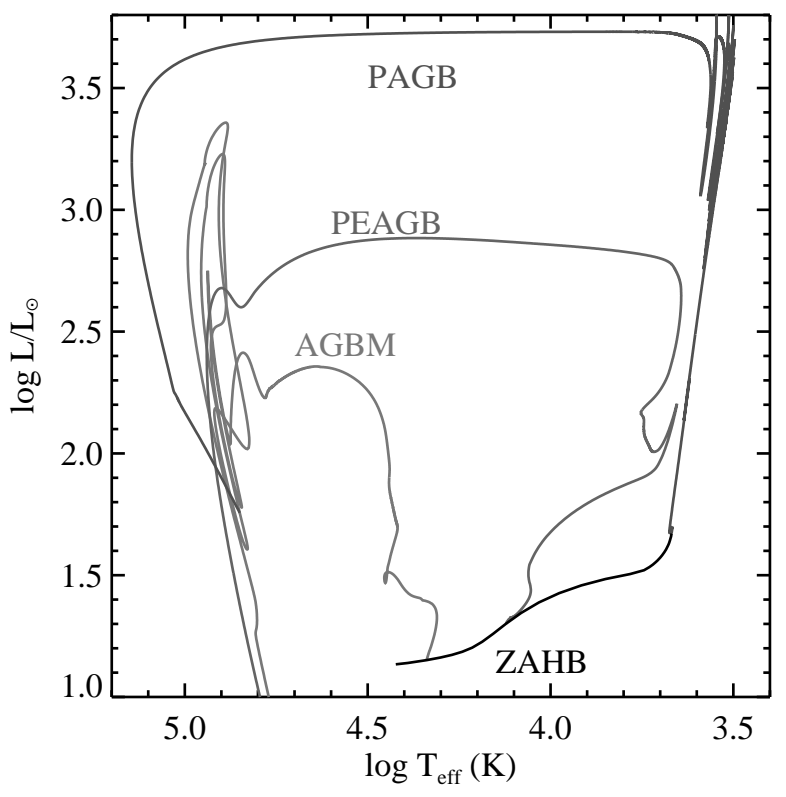

FIG. 1. - Late stages of stellar evolution for solar-metallicity low-mass stars, shown in the theoretical plane. Examples of three possible evolutionary paths from the zero-age HB (black curve; labeled) to the WD cooling curve (grey curves; labeled) are shown. In an old population, our UV CMD isolates those evolved stars at $T_{\text {eff }} \gtrsim 10,000 \mathrm{~K}$ from the far more numerous stars on the RGB, AGB, and main sequence.

lution, PAGB stars are UV-bright for only a relatively short time $\left(10^{3}-10^{4}\right.$ years $)$. For this reason most Galactic globular clusters do not currently host any known hot PAGB stars (e.g., Landsman et al. 2000).

HB stars with less massive envelopes ascend the AGB, but their envelope mass drops below $M_{\text {crit }}$ before reaching the thermally-pulsing stage. These post-early AGB (PEAGB) stars leave the AGB and cross the HR diagram at a lower luminosity than the PAGB stars, and then descend the WD cooling curve. Because $M_{\text {crit }}$ increases with decreasing luminosity, PEAGB stars will leave the AGB with a larger envelope than PAGB stars. This larger envelope, combined with the lower crossing luminosity, gives the PEAGB stars a longer UV-bright lifetime between the AGB and WD phases $\left(10^{4}\right.$ $\left.10^{5} \mathrm{yr}\right)$.

EHB stars with very little envelope mass do not ascend the AGB at all, becoming instead AGB-Manque (AGBM) or "failed AGB" stars. They leave the HB and evolve directly to higher luminosities and higher effective temperatures. Compared to the PAGB and PEAGB stars, AGBM stars are UVbright for a significantly longer time $\left(10^{6}-10^{7} \mathrm{yr}\right)$, which is within an order of magnitude of the time spent as an EHB star $\left(\sim 10^{8} \mathrm{yr}\right)$. During the AGBM phase, most of a star's luminosity comes from the helium-burning shell that forms at the end of the HB phase. As the helium-burning shell advances outward through the core, the temperature within the hydrogen-burning shell increases, until by the end of the AGBM phase the hydrogen-burning shell begins to make a non-negligible contribution to the surface luminosity. The transition from central helium burning to helium-shell burning at the end of the HB phase produces a significant luminosity gap between the EHB and AGBM stars, as will be discussed in section 3.4 .

Using the near-UV channel on the Space Telescope Imaging Spectrograph (STIS; Woodgate et al. 1998), Brown et al. (2000b) imaged the core of M32 in order to resolve the source of UV emission in elliptical galaxies. The luminosity func-
TABLE 1

OBSERVATIONS

\begin{tabular}{rrrc}
\hline & \multicolumn{3}{c}{ Exposure Time } \\
Date & Total & Low Sky & Position Angle \\
\hline 30 Jul 2001 & $13472 \mathrm{~s}$ & $9640 \mathrm{~s}$ & $-157^{\circ}$ \\
31 Jul 2001 & $13472 \mathrm{~s}$ & $9640 \mathrm{~s}$ & $-157^{\circ}$ \\
2 Aug 2001 & $13472 \mathrm{~s}$ & $9340 \mathrm{~s}$ & $-157^{\circ}$ \\
4 Aug 2001 & $13472 \mathrm{~s}$ & $9040 \mathrm{~s}$ & $-157^{\circ}$ \\
12 Oct 2002 & $13472 \mathrm{~s}$ & $10560 \mathrm{~s}$ & $+113^{\circ}$ \\
13 Oct 2002 & $13472 \mathrm{~s}$ & $10560 \mathrm{~s}$ & $+117^{\circ}$ \\
22 Oct 2002 & $13472 \mathrm{~s}$ & $10560 \mathrm{~s}$ & $+117^{\circ}$ \\
\hline
\end{tabular}

tion of stars in the field demonstrated that the bulk of the hot evolved population resides on the HB itself. However, with a single bandpass and a strongly varying bolometric correction for these evolved stars, it was difficult to disentangle the various evolutionary phases present in the image. We subsequently obtained deep far-UV STIS images of the same field, enabling the construction of a UV CMD of these populations. In this paper, we present a detailed comparison of this CMD to the expectations from stellar evolutionary theory.

\section{OBSERVATIONS AND DATA REDUCTION}

\subsection{HST Observations}

Using STIS on the Hubble Space Telescope (HST), we obtained deep far-UV images of the compact elliptical galaxy M32. As done with our earlier STIS near-UV images (Brown et al. 2000b), we targeted a position $\sim 7.7^{\prime \prime}$ south of the M32 core to allow both the bright core and the fainter regions of the galaxy to be sampled in the $25^{\prime \prime} \times 25^{\prime \prime}$ field. We also constrained the position angle of the field to maximize the overlap with our earlier near-UV images. Unfortunately, this overlap was somewhat reduced by an intrinsic offset between the field centers defined for the two STIS UV channels, such that the region with at least $80 \%$ exposure depth in both channels is approximately $21^{\prime \prime} \times 23^{\prime \prime}$ in size. Our analysis here is restricted to this region.

Our total exposure time in the strontium fluoride filter (F25SRF2) was 94,304 s, split into 7 visits of 5 orbits each (Table 1). The F25SRF2 filter blocks geocoronal emission from Lyman $\alpha$ but not O I $\lambda 1301$ or N I $\lambda 1493$ (Brown et al. 2000c). The O I emission varies strongly over an orbit, so we split each visit into a series of 47 exposures of $300 \mathrm{~s}$ each (with some of these exposure times adjusted to accommodate orbit boundaries). We did not use the STIS TIME-TAG mode, where the exposure records the location and time for every detected photon, because this mode requires careful management of the data buffer, which is difficult to do when the sky is varying so strongly. Because the STIS UV detectors are photon-counting multianode microchannel arrays (MAMAs), there is no read noise. Furthermore, the instrument can transfer data from its internal buffer to the $H S T$ data recorder during an exposure if that exposure is at least $300 \mathrm{~s}$. For these reasons, there was no penalty in obtaining a series of $300 \mathrm{~s}$ exposures instead of one long exposure per orbit, and the advantage of this approach was the ability to remove periods of high sky background due to geocoronal O I emission. Note that the MAMA detectors also register less than one count per incident cosmic-ray, so cosmic-ray rejection is not required, and breaking up an orbit into multiple exposures is not a concern, as it is for CCDs on $H S T$ (where one generally wants to keep exposures to $\sim 1000$ s or less). 


\subsection{Data Reduction}

After processing the far-UV data through the standard CALSTIS pipeline, we inspected a region free of stars in each $300 \mathrm{~s}$ exposure in order to cull those exposures with high sky background. Our threshold was 0.03 counts s${ }^{-1}$ pix $^{-1}$, with periods of low sky generally exhibiting count rates of $\sim 0.01$ counts $\mathrm{s}^{-1} \mathrm{pix}^{-1}$ and periods of high sky exhibiting count rates 10 to 100 times higher. We then coadded the low sky exposures within each visit, because they were all taken at the same position (i.e., there was no dithering during a visit). The exposure times retained for each visit are listed in Table 1. We then applied a geometric distortion correction to each visit's image, using the IRAF DRIZZLE package (Fruchter $\&$ Hook 2002). Because of inaccuracies in the STIS geometric distortion solution, the residual distortions in the resulting images did not allow satisfactory co-addition of the 2001 data with the 2002 data, given the $\sim 90^{\circ}$ roll between those datasets (see Table 1). Furthermore, the far-UV images could not be adequately registered with our earlier near-UV image (Brown et al. 2000b). Thus, using the IRAF GEOMAP task, we derived an additional distortion solution to transform the far-UV images to the near-UV reference frame, utilizing several hundred bright stars that could be identified as common to both bandpasses. We then used the IRAF GEOTRAN task to transform the far-UV images, and then coadded the far-UV data to a single image (Figure 2).

We used the DAOPHOT-II package (Stetson 1987) to construct a model point spread function (PSF) from isolated stars in the far-UV image. We then performed PSF-fitting photometry on the far-UV image, using two passes of object detection and fitting. Matching this photometry to the near-UV photometry of the same field (Brown et al. 2000b) proved to be difficult for two reasons. Due to residual small-scale differences in the geometric distortion and the variation in PSF shape with position, the inconsistency between the two bandpasses for a star's position significantly varied across the field, with the offset in each axis having an rms of $\sim 0.3$ pixels; the effects of this mis-registration was included in our analysis during the artificial star tests. Furthermore, the bandpasses are very distinct in wavelength, such that a star could be much brighter than its neighbors in one bandpass but much fainter than its neighbors in the other, to the extent that stars detected in one bandpass were often undetected in the other. In the end, we decided to perform aperture photometry ( 2 pixel radius) in the near-UV image at the positions of objects detected and fit in the far-UV image. Although the catalog of Brown et al. (2000b) used PSF-fitting photometry in the near-UV image, we did not employ PSF-fitting in the near-UV photometry for the current analysis; fixing the near-UV PSF fitting at the farUV positions caused fits to fail, due to the mis-registrations, while allowing the near-UV PSF fitting to float in position frequently caused the fits to wander onto neighboring stars, due to both the mis-registration and the large color variations from star to star. The final catalog was cleaned of severe blends by using the quality of the PSF fit in the far-UV photometry and a concentration index (i.e., comparing photometry in apertures of two distinct radii) in the near-UV photometry. The catalog was also cleaned of stars falling within the bright center of the galaxy (an irregularly-shaped region of area $9.4 \operatorname{arcsec}^{2}$ ). Although we obtained PSF-fitting photometry of 7,500 stars in the far-UV image, only 5,200 of these resulted in legitimate near-UV photometry and survived the catalog cleaning.

The photometry for each bandpass was put on an absolute magnitude scale by normalizing to aperture photometry on the brightest stars. For the near-UV photometry, this was done by comparing aperture photometry for radii of 2 and 3 pixels and then using the aperture correction of Proffitt (2003) to go from 3 pixels to infinity; for the far-UV photometry, this was done by comparing the PSF-fitting photometry to that in a 3 pixel aperture, and then applying the correction of Proffitt (2003) to go from 3 pixels to infinity. Our photometry is in the STMAG system: $m=-2.5 \log _{10} f_{\lambda}-21.1 \mathrm{mag}$, where $f_{\lambda}=e^{-}$(PHOTFLAM/EXPTIME), EXPTIME is the exposure time, and PHOTFLAM is $5.836 \times 10^{-18} \mathrm{erg} \mathrm{s}^{-1} \mathrm{~cm}^{-2}$ $\AA^{-1}\left(e^{-} \mathrm{s}^{-1}\right)^{-1}$ for the near-UV bandpass and $4.201 \times 10^{-17}$ $\operatorname{erg~s}^{-1} \mathrm{~cm}^{-2} \AA^{-1}\left(e^{-} \mathrm{s}^{-1}\right)^{-1}$ for the far-UV bandpass. These PHOTFLAM values take into account the time-dependent sensitivity of the STIS UV bandpasses, and represent the exposure-time weighted average for the dates of the observations. The STMAG system is a convenient system because it is referenced to an unambiguous flat $f_{\lambda}$ spectrum; an object with $f_{\lambda}=3.63 \times 10^{-9} \mathrm{erg} \mathrm{s}^{-1} \mathrm{~cm}^{-2} \AA^{-1}$ has a magnitude of 0 in every bandpass.

Artificial star tests were performed by inserting 400 artificial stars into the near-UV and far-UV images, blindly recovering them with the same process used to create our photometric catalog, and then repeating this process 12,500 times (for a total of 5,000,000 artificial stars). The artificial stars spanned the range $-2.5 \leq\left(m_{F U V}-m_{N U V}\right) \leq 1$ mag and $19.5 \leq m_{F U V} \leq 27 \mathrm{mag}$. No stars brighter than this range were found in the data; in our modeling, those few stars falling in brief evolutionary phases brighter than this range were assumed to have the same small scatter and nearly $100 \%$ completeness as the bright end of the artificial star tests. Although the photometric process uses aperture photometry for the near-UV measurements, a spatially-variable model of the near-UV PSF (constructed from isolated stars in the near-UV data) was used to insert artificial stars into the near-UV image. Artificial stars were inserted into the near-UV image with a small random offset from the position in the far-UV image ( $\sigma=0.3$ pixels in each axis), to approximate the effects of the small-scale residuals in the geometric distortion correction (discussed above).

Artificial stars were not distributed uniformly across the farUV and near-UV images. Instead, because object detection in our process is done on the far-UV image, the density of stars in each pass of the artificial star tests was matched to the far-UV surface-brightness profile, created by applying a ring median filter to the far-UV image. This spatially non-uniform distribution of artificial stars ensures that the resulting photometric scatter and incompleteness matrix is properly weighted to the population we are trying to model. To understand the importance of this weighting, it is useful to consider a simplified example.

Imagine a galaxy with an abrupt edge, imaged so that this edge bisects the camera's field of view. In the left-hand half of the image, there is no significant luminosity from the galaxy, and in the right-hand half of the image, there is a crowded field of stars. Assume that in the empty left-hand side of the image, an isolated EHB star could in principle be detected at a completeness of nearly $100 \%$ and measured with a photometric error of $0.02 \mathrm{mag}$, while in the crowded right-hand half of the image, such a star could be detected at $50 \%$ completeness and measured with a photometric error of $0.2 \mathrm{mag}$. Also assume that much brighter PAGB stars could be detected at nearly $100 \%$ completeness and measured with $\sim 0.01 \mathrm{mag}$ 


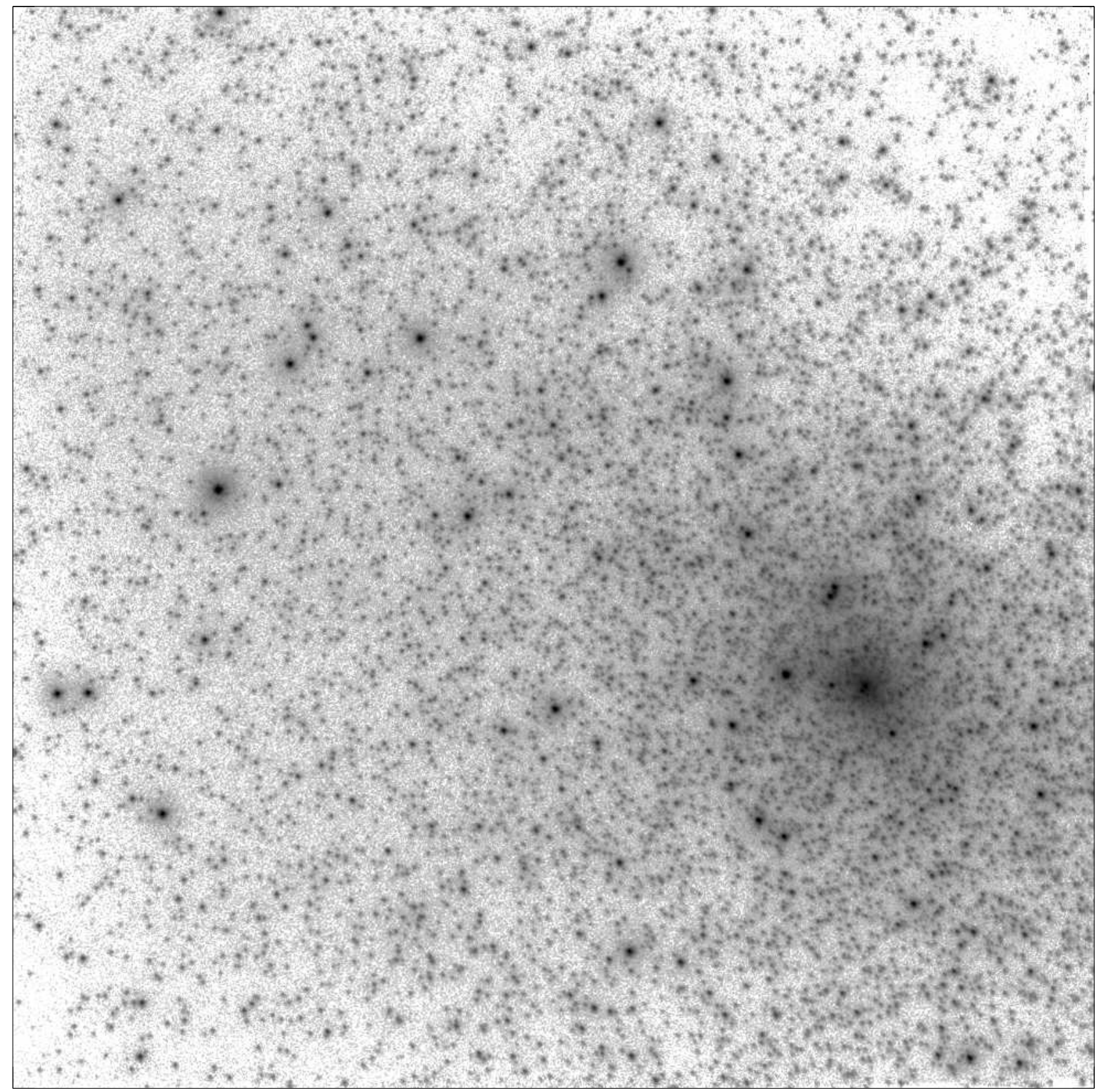

FIG. 2.- The STIS/FUV image of M32, with a logarithmic stretch and cropped to $24^{\prime \prime} \times 24^{\prime \prime}$.

photometric errors anywhere in the image. If one performed artificial star tests on such an image with a uniform distribution of stars, those tests would give an erroneous completeness correction and imply an erroneous ratio of EHB to PAGB stars in the field. Specifically, one would incorrectly find a completeness of $75 \%$ and a photometric error of 0.08 mag for the EHB stars. In reality, the population being studied only exists in the right-hand side of the image, where EHB stars have lower completeness and larger photometric errors. Instead, by distributing the artificial stars so that their density follows the luminosity in the image, one recovers an accurate measure of the completeness and photometric scatter in the entire population. Alternatively, one could uniformly distribute the artificial star tests and weight the results by the luminosity profile in the image.

The knowledge of the completeness and photometric scatter can then be applied to the modeling in different ways. One can attempt to correct the data before comparison to the models, but it is difficult to deconvolve the photometric scatter. Instead, in our analysis here, we work in the opposite sense: we apply the incompleteness and photometric scatter to the models to create simulations, and then compare to the uncorrected data.

\subsection{The Color-Magnitude Diagram}

We show the UV CMD of M32 in Figure 3. The most obvious feature of the CMD is the presence of a hot $\mathrm{HB}$ population. The existence of these stars was inferred by Brown et al. (2000b) from the luminosity function in a single near-UV bandpass; with the present CMD, the detection of an EHB population in M32 is unequivocal. The other striking features of this CMD are the small number of UV-bright stars above the $\mathrm{HB}$ and the lack of a luminosity gap between the EHB and these UV-bright stars. We will explore these aspects of 


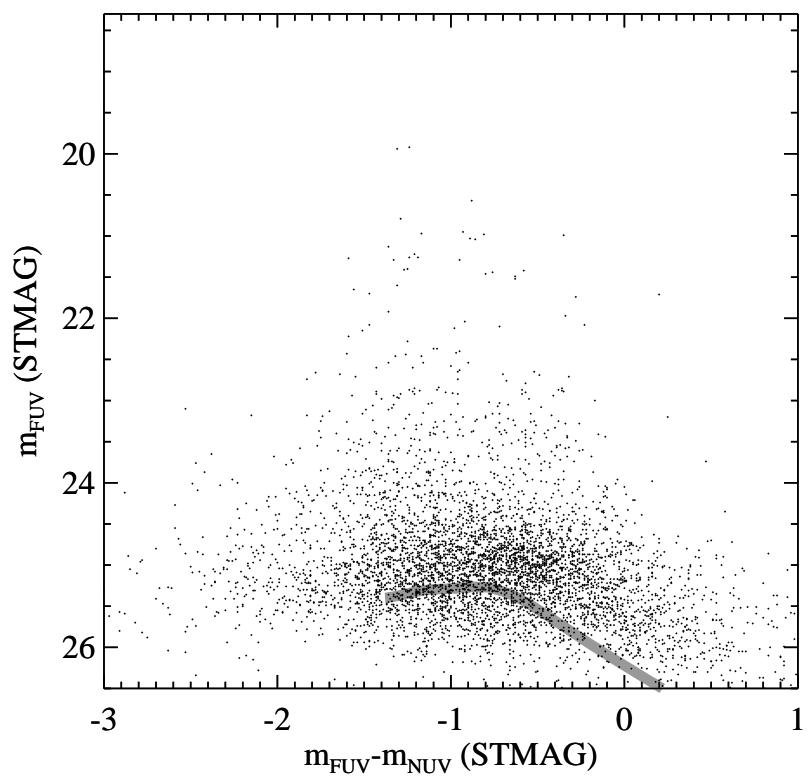

FIG. 3.- The UV CMD of M32. The EHB and UV-bright post-HB stars are clearly resolved. A solar-metallicity zero-age $\mathrm{HB}$ is plotted for reference (grey curve).

the CMD in the modeling below. Note that in the region of M32 spanned by our UV catalog (i.e., the sky sampled by both the near-UV and far-UV images but excluding the 9.4 $\operatorname{arcsec}^{2}$ region in the M32 core), the optical surface brightness of M31 is $<<1 \%$ that of M32 (Walterbos \& Kennicutt 1987), so contamination from M31 stars should be negligible.

\section{EVOLUTIONARY SEQUENCES}

\subsection{HB and post-HB Tracks}

We have calculated a grid of $\mathrm{HB}$ and post-HB evolutionary tracks with a variety of abundances appropriate for analyzing the UV CMD of M32. These tracks span a wide range of HB masses from the hot end of the EHB to the red $\mathrm{HB}$ with a fine mass spacing, in order to capture the full range of post-HB behavior. For most tracks, the evolution was followed through the HB phase using standard algorithms for convective overshooting and semiconvection (Robertson \& Faulkner 1972) and then through the post-HB phase until the models reached a faint luminosity on the WD cooling curve. However, a small subset of our models encountered a final helium-shell flash while descending the WD cooling curve. The calculations for these models were stopped if the resulting flash convection extended into the hydrogen envelope.

For each composition we first obtained a zero-age HB (ZAHB) model at the red end of the HB by evolving a model from the zero-age main sequence, up the RGB, and then through the helium-core flash at the tip of the RGB. The initial mass for these sequences was adjusted for each composition to give an age of $13 \mathrm{Gyr}$ at the ZAHB phase. This assumed age for the HB stars in M32 is not, however, important for our analysis, since the helium-core mass of a ZAHB model is only weakly dependent on the initial mass of the star. Assuming a younger age, and hence larger main sequence mass, would simply yield a slightly redder ZAHB model with a larger envelope mass but the same helium-core mass. We then produced the hotter ZAHB models needed for our HB and post$\mathrm{HB}$ tracks by removing mass from the envelope of our red ZAHB model.

We computed evolutionary tracks for three values of the metallicity: $[\mathrm{Fe} / \mathrm{H}]=0.0,-0.25$ and -0.45 , corresponding to heavy-element abundances $Z=0.01716,0.01$, and 0.006 , respectively. Scaled solar abundances were assumed for these metallicities with no enhancement of the $\alpha$ elements, i.e., $[\alpha / \mathrm{Fe}]=0.0$. These metallicities cover the range spanned by the bulk of the M32 population (see Worthey et al. 2004), with $[\mathrm{Fe} / \mathrm{H}]=-0.25$ lying near the peak of the M32 metallicity distribution. The tracks with $[\mathrm{Fe} / \mathrm{H}]=0.0$ were calibrated by adjusting the heavy-element abundance $Z$ to 0.01716 , the helium abundance $Y$ to 0.2798 , and the mixing-length ratio $\alpha$ to 1.8452 , in order to match the observed solar luminosity, radius, and $Z / X$ ratio at an age of $4.6 \mathrm{Gyr}$. The helium abundance of these solar metallicity models increased to 0.3003 during the first dredge-up on the RGB, and this therefore represents the envelope helium abundance during the subsequent $\mathrm{HB}$ and post-HB evolution. The main-sequence helium abundance of the $[\mathrm{Fe} / \mathrm{H}]=-0.25$ and -0.45 sequences was set at $Y=0.23$, which increased to 0.2518 and 0.2496 , respectively, during the first dredge-up. We also calculated a set of EHB models (including their post-HB progeny) with enhanced helium abundances of $Y=0.28,0.33,0.38$, and 0.43 in order to explore the strong effect of $Y$ on the HB and post-HB evolution.

Mass loss was included during the AGB phase using the Reimers (1975) formalism, which is parameterized by the mass-loss parameter $\eta_{R}$. We calculated AGB tracks for $\eta_{R}$ values of both 0.4 and 1.0. In $\S 3.3$, we will show that both the lifetime and mean luminosity of a PAGB star within the UVbright region of Figure 3 depend primarily on the final mass of the star. Thus a bluer HB star that evolves up the AGB with a smaller mass loss can have the same UV-bright lifetime and mean luminosity as a redder HB star that evolves with a larger mass loss if the final PAGB masses are the same. Our use of different $\eta_{R}$ values was simply a means for producing PAGB stars over a range in mass. It was not intended to represent the actual mass loss process along the AGB. Finally we note that there was no mass loss in the AGBM sequences, which do not ascend the AGB at all.

\subsection{Translation to Observed Parameters}

We translate our HB and post-HB tracks (and others from the literature) into observed magnitudes $\left(m_{F U V}\right.$ and $\left.m_{N U V}\right)$ by folding synthetic spectra through the STIS effective area curves. We use the Castelli \& Kurucz (2003) grid of synthetic spectra for $T_{\text {eff }} \leq 50,000 \mathrm{~K}$ and blackbody spectra for hotter stars. The grid of synthetic spectra is interpolated in effective temperature, surface gravity, and metallicity, assuming scaled solar abundances (no alpha enhancement). Because the synthetic spectra cannot account for any helium enhancement, we only match the spectra to the $Z$ value of our evolutionary tracks, even when $Y$ is enhanced. We assume that M32 is at the same distance as M31 (770 kpc; Freedman \& Madore 1991), and we generally assume a foreground extinction of $E(B-V)=0.08 \mathrm{mag}$ (Schlegel et al. 1998) and the mean Galactic extinction law of Fitzpatrick (1999). In addition, we also consider a small dispersion in reddening for the sightlines to the stars in our catalog. Potential sources of reddening variation in the field include Galactic foreground, M31 disk (if M32 lies slightly behind the M31 disk), and dust intrinsic to M32 itself (which should be minimal; see Corbin et al. 2001). At this time, it is unclear if M32 lies behind the M31 disk (see Worthey et al. 2004) or in front of it (see Ford et al. 1978; Paulin-Henriksson et al. 2002). 


\subsection{Post-HB Evolution}

As described in $\S 1$, the post-HB evolutionary path of a star is driven by its envelope mass on the $\mathrm{HB}$. At the one extreme, red HB stars with large envelopes give rise to PAGB stars, while at the other extreme, EHB stars with small envelopes give rise to AGBM stars. Regardless of the evolutionary path, all stars leaving the $\mathrm{HB}$ will spend at least some time as a hot post-HB star within the UV-bright region of Figure 3. For the purposes of the discussion here, we define a "hot post-HB star" as a star residing at $\left(m_{F U V}-m_{N U V}\right)<0$ mag and $0.5 \mathrm{mag}$ brighter than the end of the core helium-burning phase for a solar-metallicity HB (see Figure 4). The luminosity and duration of this hot post-HB phase depend on a star's final mass on the WD cooling curve. A star with high mass will evolve through the PAGB phase at high luminosities on a short timescale (thousands of years), while a star with low mass will evolve through the AGBM phase at low luminosity on a long timescale (millions of years).

In order to explore these properties in more detail, we have calculated both the time spent within the "hot post-HB star" region of Figure 4 and the mean bolometric luminosity within this region for each of our post-HB evolutionary tracks. The results for our solar-metallicity tracks, given in Figure 5, show a tight correlation from the faint, but long-lived, AGBM tracks to the bright, but short-lived, PAGB tracks. Our tracks with AGB mass-loss rates of $\eta_{R}=0.4$ and $\eta_{R}=1.0$ fall on the same trend, indicating that the correlation in Figure 5 is insensitive to the details of the AGB mass-loss process. For comparison, we also plot the same results for the solar-metallicity tracks of Dorman et al. (1993; hereafter DRO93), Yi et al. (1997; hereafter YDK97), and Vassiliadis \& Wood (1994; hereafter VW94), and in all of these cases we find good agreement with our models. The H-burning and He-burning PAGB tracks of VW94 in Figure 5 correspond to main sequence turnoff masses of 1.0, 1.5, and 2.0 $M_{\odot}$. With the mass-loss assumptions of VW94, these main-sequence masses produce PAGB stars having masses of $0.57-0.63 M_{\odot}$. The $0.633 M_{\odot} \mathrm{H}-$ burning PAGB track of VW94 that evolved from a $2 M_{\odot}$ main-sequence star is similar to our own $\sim 0.6 M_{\odot}$ tracks that evolved from a $1 M_{\odot}$ main-sequence star, further suggesting that the properties of a PAGB star are mainly governed by the star's final mass.

Because variations in metallicity will be one of the parameters that we will later explore in $\$ 4$ when trying to model the M32 UV CMD, it is worth determining whether the correlation shown in Figure 5 depends upon metallicity. In Figure 6 , we show a similar plot, but limited to our own tracks at three values of $[\mathrm{Fe} / \mathrm{H}]:+0.0$ (solar), -0.25 , and -0.45 . The same tight correlation between lifetime and luminosity found in Figure 5 persists for all of these metallicities.

Figure 5 strongly indicates, but does not prove, that the duration of the hot post-HB phase and its time-averaged bolometric luminosity are each determined by a star's final mass. In order to examine this point more closely, we plot each of these quantities separately in Figure 7 as a function of the final mass for both our tracks and those of DRO93, YDK97, and VW94. Again we see that the long-lived AGBM tracks are clearly separated from the shorter-lived PEAGB and PAGB tracks. The DRO93 tracks deviate towards shorter durations but brighter luminosities for the hot post-HB phase. Except for this deviation all of the other tracks in Figure 7 agree well. We conclude that the properties of our tracks within the hot post-HB star region are primarily determined by the final mass and are not dependent on the details of the prior mass loss on the AGB.

In order to utilize our post-HB evolutionary tracks in the analysis of the UV CMD of M32, we need to replace the theoretical parameters in Figure 5 with the corresponding observable parameters. Replacing the bolometric luminosity with the far-UV magnitude is straightforward, as discussed in $§ 3.2$. However, replacing the duration of the hot post-HB phase with the expected number of hot post-HB stars for a given evolutionary track requires a more detailed explanation.

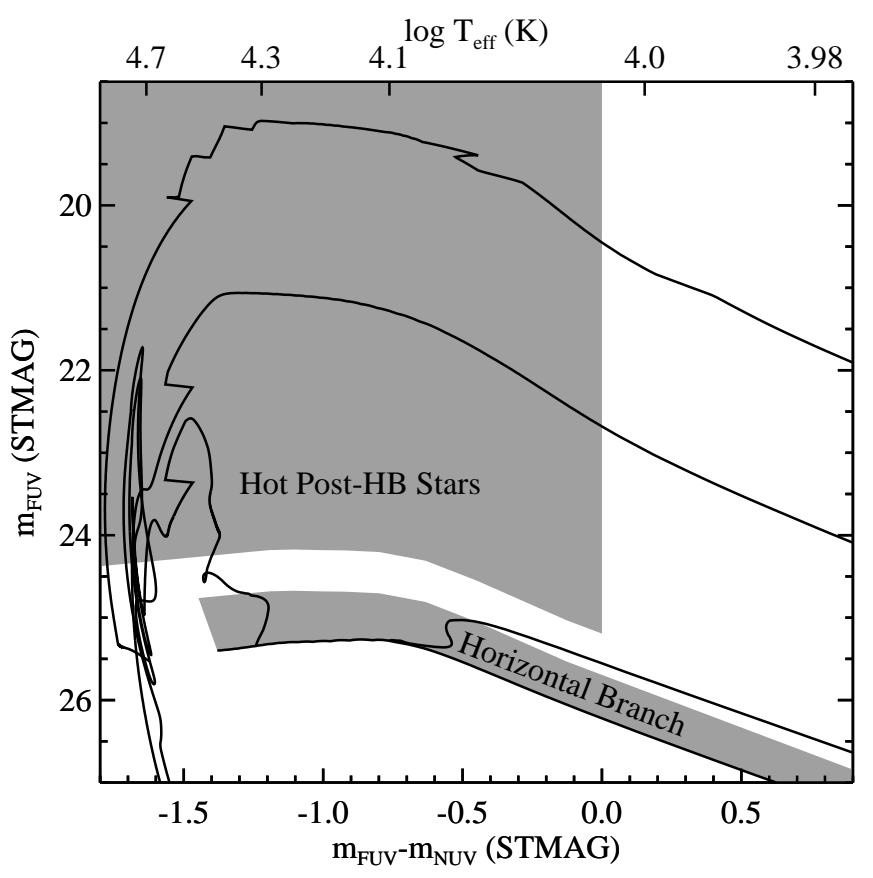

FIG. 4.- The evolutionary paths shown in Figure 1, but transformed to the STIS UV bandpasses assuming a distance of $770 \mathrm{kpc}$ (Freedman \& Madore 1991) and $E(B-V)=0.08 \mathrm{mag}$ (Schlegel et al. 1998). The region used to define the "hot post-HB stars" in the present discussion is highlighted (grey shading; labeled). The relation between $T_{\mathrm{eff}}$ and color on the x-axis assumes $\log g=5$ and $[\mathrm{Fe} / \mathrm{H}]=0$.

The number of stars evolving along any particular post-HB track is constrained by the stellar evolutionary flux (SEF) of the population, i.e., by the rate at which the stars in the population are leaving any evolutionary phase. In general, the number $N_{j}$ of stars in any particular evolutionary phase $j$ is the product of the duration of that phase $t_{j}$, the specific evolutionary flux of the population $B(t)$ (i.e., the stellar death rate per unit luminosity), and the total bolometric luminosity $L_{T}$ of the population (Greggio \& Renzini 1990; Renzini 1998):

$$
N_{j}=B(t) L_{T} t_{j}
$$

$B(t)$ depends weakly upon age and metallicity and has a value

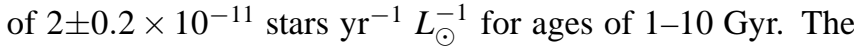
$\mathrm{SEF}$ is then given by $S E F \equiv N_{j} / t_{j} \equiv B(t) L_{T}$. The expected number of hot post-HB stars in the population then follows from the SEF and the duration $t_{j}$ of the hot post-HB phase.

Our UV catalog covers an area of approximately $21^{\prime \prime} \times 23^{\prime \prime}$, excluding a $9.4 \operatorname{arcsec}^{2}$ area in the center of the galaxy. Based on WFPC2/F555W images of this area, we find its optical luminosity is $V=9.97 \mathrm{mag}$. Given a foreground extinction of $E(B-V)=0.08 \mathrm{mag}$ (Schlegel et al. 1998) and a distance of $770 \mathrm{kpc}$ (Freedman \& Madore 1991), we obtain 


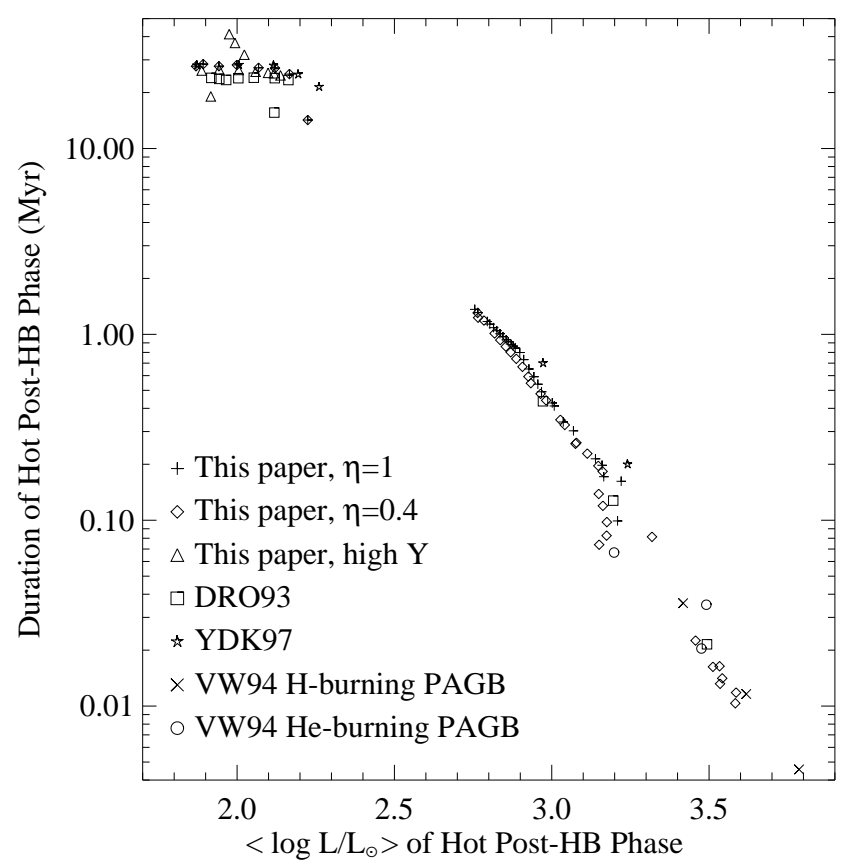

FIG. 5.- The time spent as a hot post-HB star as a function of the timeaveraged luminosity in this phase. The AGBM stars are relatively long-lived and faint, while the PAGB stars are relatively short-lived and bright. The gap in the distribution at $\log L \approx 2.5 L \odot$ separates the AGBM stars from the PEAGB stars. Other evolutionary tracks from the literature are also shown, giving good agreement with our own tracks.

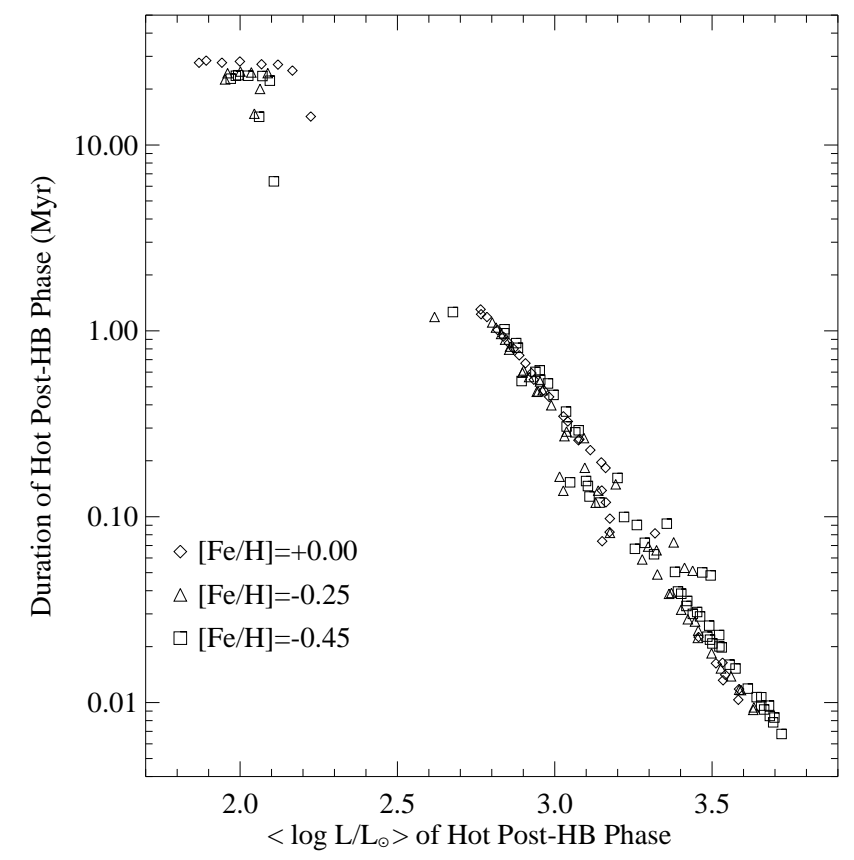

FIG. 6.- The time spent as a hot post-HB star as a function of the timeaveraged luminosity in this phase, comparing our tracks at three different metallicities. The tight correlation shown in Figure 5 persists even when the metallicity is varied.

$M_{V}=-14.71 \mathrm{mag}$. If we assume an age of $8 \mathrm{Gyr}$ for a population of nearly solar metallicity, the bolometric correction is $-0.875 \mathrm{mag}$ (Worthey 1994), with an uncertainty of $\sim 0.1$ mag for ages of 5-12 Gyr. The bolometric luminosity is thus $1.36 \times 10^{8} L_{\odot}$, which gives an SEF of $3.0 \times 10^{-3} \mathrm{star}$
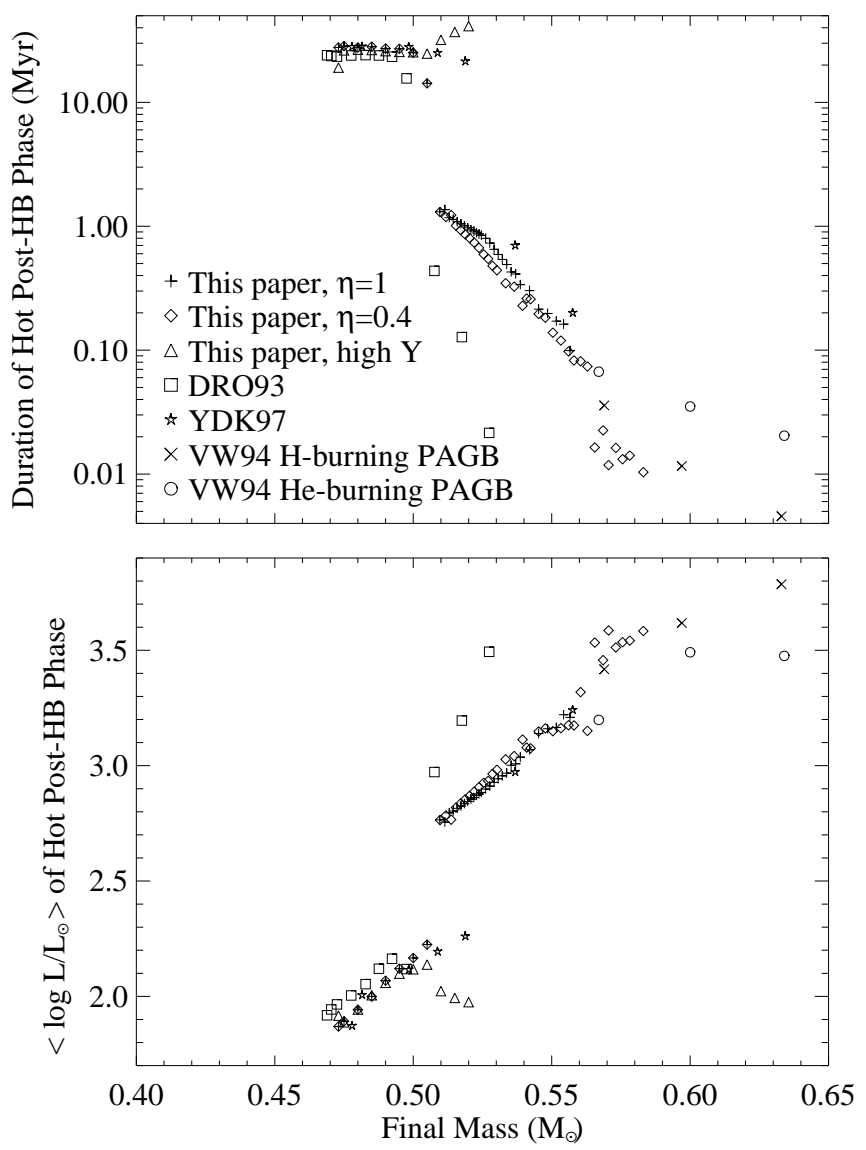

FIG. 7.- Top panel: The time spent as a hot post-HB star as a function of the final mass on the WD cooling curve, shown for our own evolutionary sequences and other models from the literature. Bottom panel: The timeaveraged luminosity in the hot post-HB phase as a function of the final mass on the WD cooling curve.

$\mathrm{yr}^{-1}$.

In Figure 8, we transform the theoretical parameters in Figure 5 into the observational plane (number of stars vs. $<m_{F U V}>$ ). The expected number of stars evolving along each track has been normalized to the total number of stars leaving the main sequence in our cataloged region $\left(3.0 \times 10^{-3}\right.$ star $\left.\mathrm{yr}^{-1}\right)$. In other words, Figure 8 shows for each evolutionary track the number of hot post-HB stars that would be present in this region if the entire population evolved along that particular track. In reality, there is a dispersion in the HB mass resulting from a range in mass loss on the RGB, which leads to a dispersion in the post-HB behavior. Thus the total SEF should be distributed amongst the available tracks, as we will do in our CMD simulations in $\S 4$.

There is a clear progression from the long-lived AGBM tracks that would yield $\sim 10^{5} \mathrm{UV}$-bright stars in our field to the short-lived PAGB tracks that would yield $\sim 10$ UV-bright stars in our field. There is more scatter in Figure 8 than Figure 5, due to the large bolometric correction at high effective temperature, even in these UV bandpasses.

Our CMD simulations will include the effects of completeness and photometric scatter, but putting those effects aside for the moment, it is clear from Figure 8 that few of the stars in M32 are evolving along AGBM tracks. If every star leaving the main sequence evolved along such tracks, our cata$\log$ would include $\sim 10^{5}$ AGBM stars, with approximately ten times as many EHB progenitors - far more AGBM and EHB 


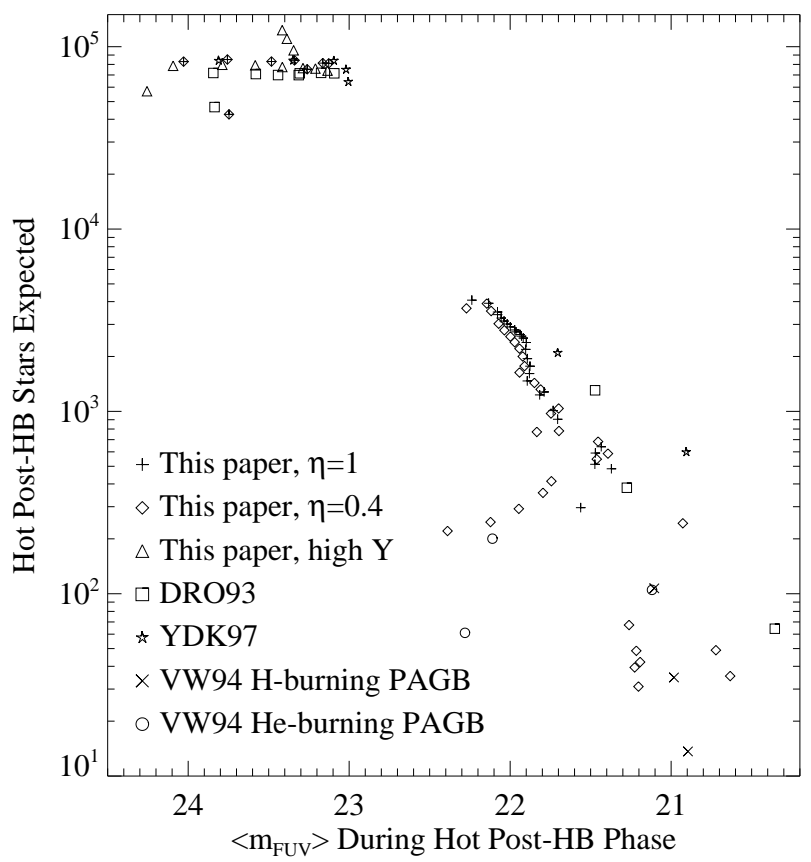

FIG. 8.- The same as in Figure 5, but shown in terms of observable parameters. For each evolutionary track, the expected number of hot post$\mathrm{HB}$ stars assumes that all of the SEF in our observed region of M32 evolves along that track. It is thus an upper limit on the stars that can be following that track, because in reality the SEF will be distributed over a range of $\mathrm{HB}$ tracks. Although there is still a clear relationship between the number of expected stars and their mean far-UV luminosity, the scatter is larger here than in Figure 5 due to the large variation in bolometric correction across the hot post-HB region.

stars than actually observed in our UV CMD of M32. Such a large number of AGBM and EHB stars would also produce a much stronger UV-upturn in M32 than observed. Indeed, the observed UV upturn in M32 requires that only $\approx 2 \%$ of the HB stars lie on the EHB. This discrepancy implies that most of the population must evolve from the red HB to become either PEAGB or PAGB stars, but there is a problem with those tracks, too: the brightest PAGB tracks produce tens of stars brighter than any seen in our image, while the fainter PAGB and PEAGB tracks produce hundreds of stars at magnitudes where we detect only a few such stars.

We conclude that current stellar evolutionary models predict many more hot post-HB stars in M32 than are actually observed regardless of how these stars evolve from the HB to the WD cooling curve. We will explore this discrepancy further in $\S 4$, where we will use CMD simulations, incorporating the photometric scatter and incompleteness from the data, to analyze the M32 UV CMD.

\subsection{Theoretical Luminosity Gap}

Virtually all of the helium-burning luminosity in an $\mathrm{HB}$ star is produced within the innermost $\sim 0.04 M_{\odot}$ of the core. The energy from this region is carried outward by convection through the convective core and then by radiation through the semiconvective zone and the outer radiative region of the core. With time the central helium abundance $\left(Y_{c}\right)$ decreases. However, due to the high temperature dependence of the heliumburning reactions $\left(\propto T^{35}\right)$ an HB star is able to compensate for this decrease in $Y_{c}$ by a small increase in the central temperature. Indeed the helium-burning luminosity actually increases throughout the $\mathrm{HB}$ phase, as the helium in the core is converted to carbon and oxygen and the convective core and semiconvective zone grow in extent. This increase in the helium-burning luminosity is responsible for the luminosity width of the EHB, where helium burning is the dominant energy source.

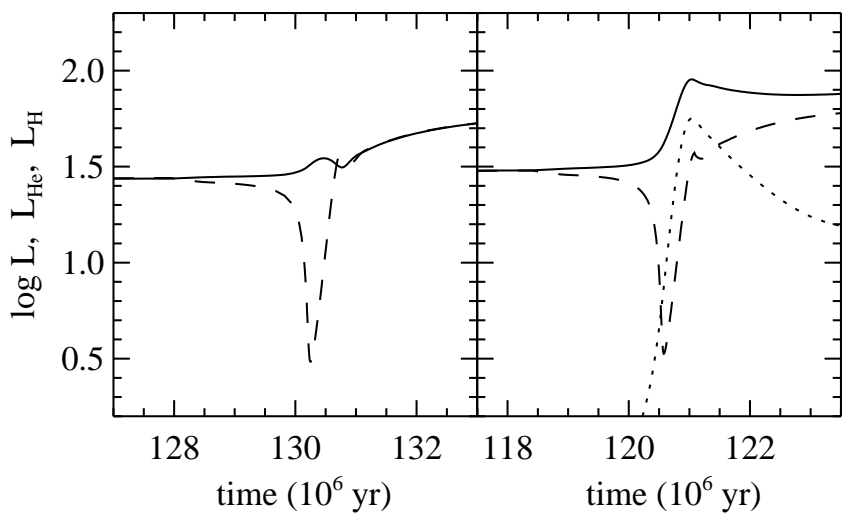

FIG. 9.- Time dependence of the surface luminosity (solid curve), heliumburning luminosity (dashed curve), and hydrogen-burning luminosity (dotted curve) at the end of the HB phase for two EHB stars. The left panel refers to a star at the hot end of the EHB with a ZAHB effective temperature of log $T_{\text {eff }}=4.46$, while the right panel refers to a star at the cool end of the EHB with a ZAHB effective temperature of $\log T_{\text {eff }}=4.23$. The abscissa gives the time elapsed since the ZAHB phase in units of $10^{6} \mathrm{yr}$. All luminosities are in solar units.

At the end of the HB phase, $Y_{c}$ goes to zero and helium burning ceases at the center. This leads to an abrupt drop in the helium-burning luminosity and to the disappearance of the convective core. In response to the loss of this major energy source, the core of an HB star is forced to contract, as the star tries to maintain its surface luminosity through the release of gravitational potential energy. This contraction heats the layers within the former semiconvective zone until the temperature within these layers rises sufficiently for helium burning to begin in a shell. This sequence of events is illustrated in the left panel of Figure 9, where we show the time dependence of the surface luminosity and the helium-burning luminosity at the end of the HB phase for a star at the hot end of the EHB. The drop in the helium-burning luminosity at the end of the $\mathrm{HB}$ phase reaches a minimum at time $\mathrm{t}=130.2 \mathrm{Myr}$. Following this minimum the helium-burning luminosity recovers as the newly formed helium-burning shell stabilizes and the star returns to thermal equilibrium. Note that the transition from central helium to helium-shell burning is marked by an increase in the surface luminosity. Hydrogen burning during this phase is completely negligible due to the very small envelope mass of this star $\left(6 \times 10^{-4} M_{\odot}\right)$.

The same sequence of events occurs in cooler HB stars but with one important difference. The right panel of Figure 9 shows the time dependence of the surface, helium-burning, and hydrogen-burning luminosities at the end of the HB phase for a star near the red end of the EHB. Again we see the drop in the helium-burning luminosity associated with the transition from central helium to helium-shell burning. The contraction of the core during this transition also raises the temperature within the hydrogen-burning shell. Due to the larger envelope mass $\left(\sim 0.02 M_{\odot}\right)$ of the star in the right panel of Figure 9, the hydrogen-burning shell turns on during this contraction and becomes a major energy source for the star. This leads to a sudden increase in the surface luminosity which should appear observationally as a luminosity gap between 
the EHB and post-EHB stars. Such a luminosity gap is actually observed in globular clusters with cooler HB stars where the base of the AGB appears as a clump well separated from the HB. This AGB clump has been detected in 47 Tuc (Hesser et al. 1987; Montegriffo et al. 1995) as well as in a number of other clusters (Ferraro et al. 1999). In a study of the early AGB evolution in globular clusters, Cassisi et al. (2001) found that the size of the predicted luminosity gap between the HB and AGB is not affected by the use of updated input physics in their stellar models.

The properties of this luminosity gap are illustrated in the top panel of Figure 10 for $6 \mathrm{EHB}$ and post-EHB evolutionary sequences with $Z=0.01$ and $Y=0.23$. We plot each track in this panel by a series of points spaced every $10^{6} \mathrm{yr}$ during the evolution in order to show where one would expect to find stars in an actual CMD. The hottest track in this panel lies at the hot end of the EHB with a ZAHB effective temperature of $\log T_{\text {eff }}=4.46$. The masses of the cooler EHB tracks increase with an increment of $0.005 M_{\odot}$. The tracks in this panel cover the mass range that yields hot post-HB stars. The luminosity gap predicted by our models can be clearly seen between the EHB and post-EHB phases.

The size of the luminosity gap depends on how strongly the hydrogen-burning shell turns on at the end of the HB phase and hence on the envelope mass of the star. Because a heliumrich EHB star has a larger envelope mass at a given effective temperature, one would expect a larger luminosity gap at higher helium abundances. This expectation is confirmed in the middle and bottom panels of Figure 10, where we plot the EHB and post-EHB tracks for the same value of $Z(=0.01)$ but for $Y$ values of 0.28 and 0.33 . As in the top panel, each track is again plotted as a series of points spaced every $10^{6} \mathrm{yr}$ during the evolution, and the mass increment between successive tracks is again $0.005 M_{\odot}$. Besides the larger luminosity gap for the helium-rich compositions, we also note that the mass range of EHB stars that evolve into hot post-EHB stars is considerably larger than in the top panel of Figure 10.

The luminosity gap shown in Figure 10 is a consequence of the changes that occur in the basic structure of a star at the end of the EHB phase and therefore should be a robust prediction of the theoretical models. In the following section we will use CMD simulations derived from our evolutionary tracks to compare this predicted luminosity gap with the observed UV CMD of M32.

\section{CMD SIMULATIONS}

\subsection{Number of UV-Bright Stars}

Our initial simulations are simple models that demonstrate the issues discussed in previous sections. At times we will follow these fits with standard numerical algorithms for CMD fitting, but the utility of such techniques is hampered by the fundamental discrepancies between the models and the data. We start with solar-abundance models at a distance of $770 \mathrm{kpc}$ (Freedman \& Madore 1990) and an extinction of $E(B-V)=$ 0.08 mag. First we vary the distribution of mass on the HB. In Figure 11, we show three different distributions of mass: a flat distribution, a red $\mathrm{HB}$ with an extension to the blue, and a bimodal HB (two Gaussians). In each simulation, the total number of HB stars is consistent with the SEF of $3.0 \times$ $10^{-3}$ stars $\mathrm{yr}^{-1}$, but the number of stars that appear in the UV varies dramatically. The simulated CMDs are shown in the left-hand panels, while the corresponding mass distributions are shown in the right-hand panels. In Figure 12, we show a comparison between the observed far-UV luminosity function

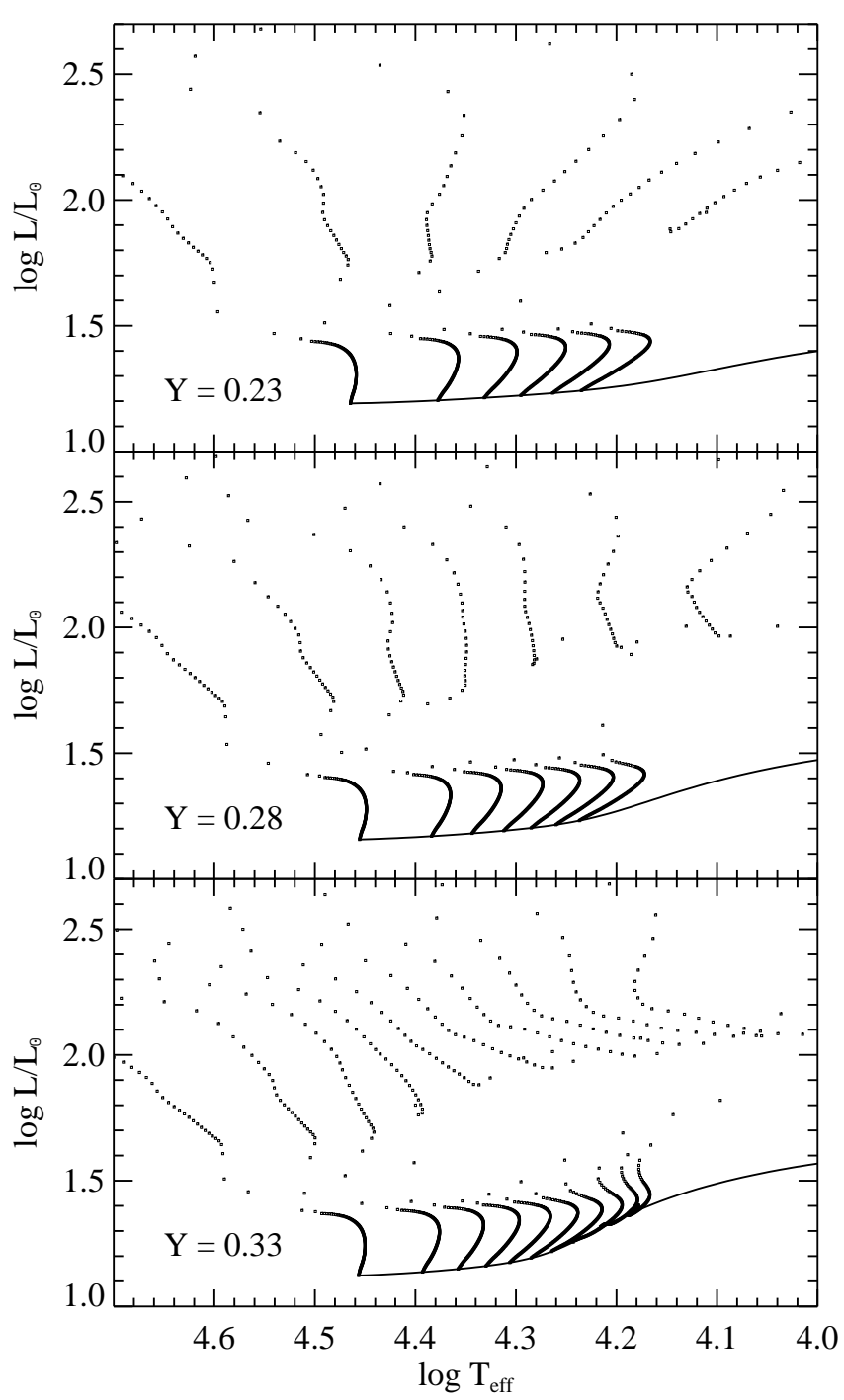

FIG. 10. - EHB and post-EHB evolutionary tracks for $Z=0.01$ and $Y=$ 0.23 (top panel), 0.28 (middle panel), and 0.33 (bottom panel). Each track is plotted by a series of points spaced every $10^{6} \mathrm{yr}$ during the evolution. The hottest track in each panel lies at the hot end of the EHB. The mass spacing between the tracks in each panel is $0.005 M_{\odot}$. The solid curves represent the ZAHB for each composition.

and the luminosity function derived for each of the simulated CMDs.

The flat distribution (top panels in Figures 11 and 12) obviously does not work - it is included only as a starting point for the discussion. Note that this is a flat distribution in mass, not a flat distribution in effective temperature - the bulk of the HB stars in this distribution are on the red HB. Far too many stars are populating the EHB and post-HB phases. To reproduce the observed CMD, we clearly need only a small minority of stars at low envelope mass.

The extended HB does much better, but is still problematic. This simulation was created using a Gaussian distribution of mass centered on the red end of the HB, with the dispersion in mass tuned to reproduce the number of EHB stars observed in our M31 CMD, with $22 \%$ of the HB stars lying on the EHB. However, there are far too many UV-bright stars above the $\mathrm{HB}$, compared to observations. Thus there are too many stars on those PEAGB and PAGB tracks that are relatively long- 

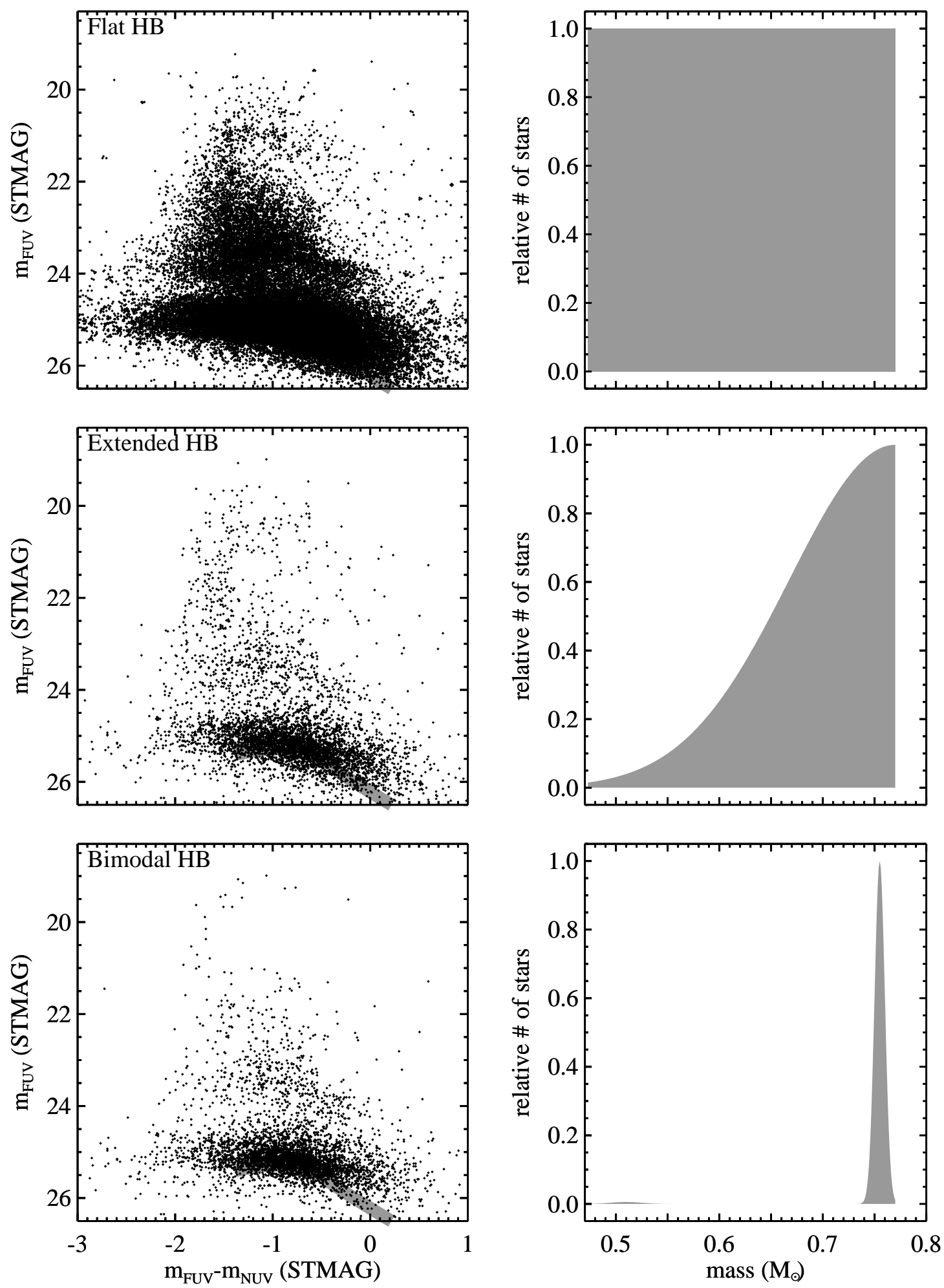

FIG. 11.- Simulated CMDs (left panels) corresponding to arbitrary distributions of mass on the HB (right panels). A flat distribution of mass on the HB (top panels) produces far too many stars on and above the EHB. A predominantly red HB with a Gaussian extension to the blue (middle panels) can be tuned to approximate the observed number of EHB stars, but predicts far too many UV-bright stars above the EHB. A bimodal distribution of HB mass (bottom panels) can reduce the predicted number of UV-bright stars above the EHB, but it is still difficult to reproduce both the observed number of these stars and their luminosity (see text). Also, the gap between the EHB and the AGBM is much more pronounced in the simulations than in the data. 

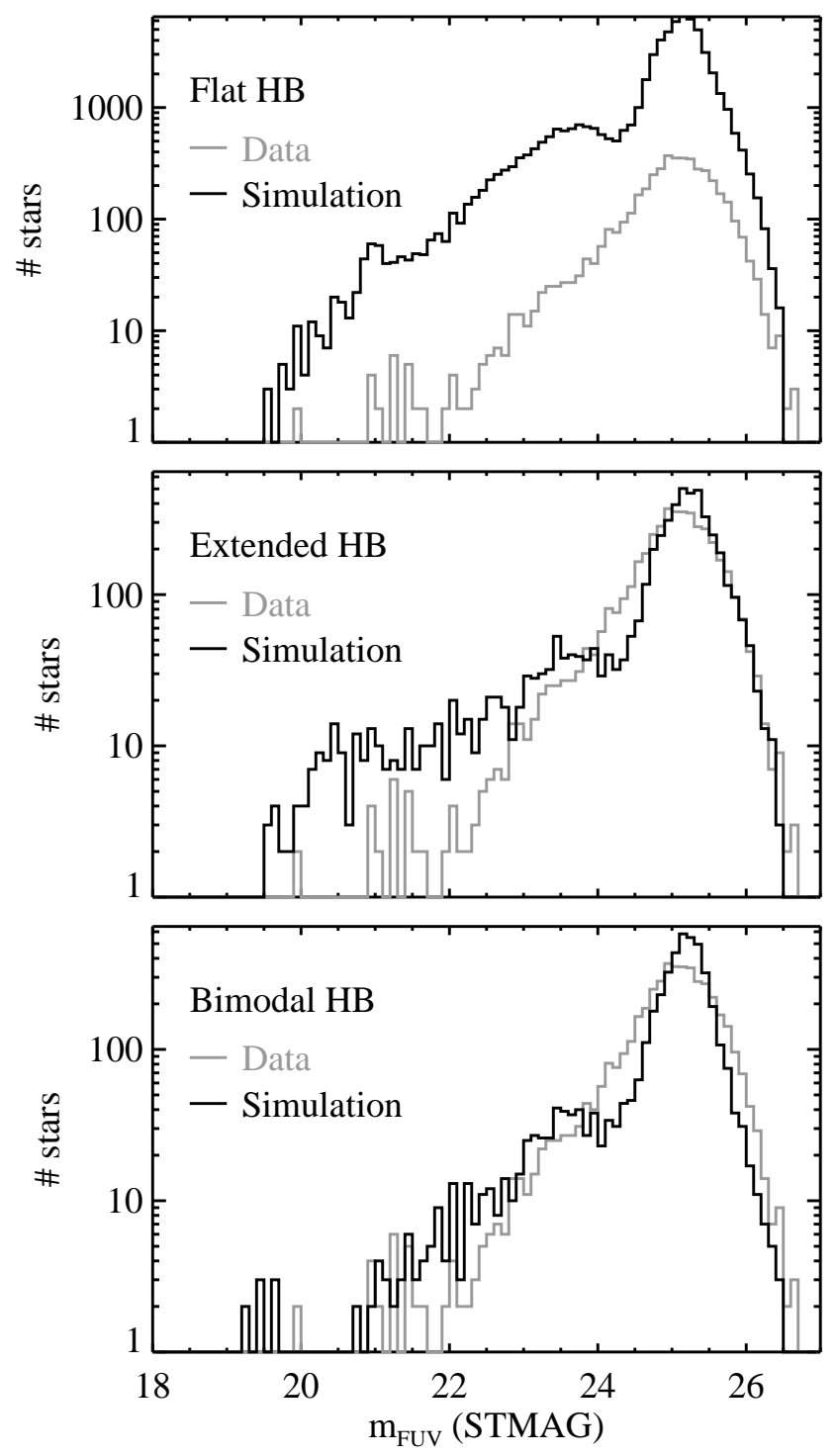

FIG. 12.- Luminosity functions for each of the simulated CMDs from Figure 11 (black histograms) compared to the observed far-UV luminosity function (grey histogram). The luminosity function spans the color range $-2 \leq\left(m_{F U V}-m_{N U V}\right) \leq 0$ mag. Instead of correcting the observed luminosity function for completeness, the model luminosity functions include both the completeness and photometric scatter, determined from artificial star tests. The luminosity gap between the EHB and AGBM stars is very clear in the simulated CMDs, but not obviously present in the observed population.

lived, with intermediate $\mathrm{HB}$ masses (between those with the lowest mass, which evolve into long-lived AGBM stars, and those with the highest mass, which evolve into very shortlived PAGB stars).

The bimodal HB does even better, by transferring weight from the PEAGB-producing tracks to the PAGB-producing tracks, thus reducing the number of UV-bright stars above the $\mathrm{EHB}$, but there are still several discrepancies. There are more PAGB stars than observed, and they extend to brighter luminosities. Furthermore, this simulation predicts a pronounced luminosity gap between the EHB and post-HB stars, which does not appear in the observed population. The luminosity width of the simulated HB is also much narrower than the observed $\mathrm{HB}$.

We next attempt to improve the fit to the observed CMD by letting the weights of the EHB tracks float freely (in- stead of fixing them as in the bimodal Gaussian distribution of Figure 11). Specifically, we fit the data via minimization of a Maximum Likelihood statistic, using an amoeba algorithm to drive the weights of each evolutionary track, and binning the data and models by $0.05 \mathrm{mag}$ in both $m_{F U V}-m_{N U V}$ color and $m_{F U V}$ luminosity. In this fitting, we enforced the constraint that the total weight in all tracks must equal the SEF of $3 \times 10^{-3}$ stars $\mathrm{yr}^{-1}$. The weights for the tracks on the EHB were allowed to float freely within this constraint, and any remaining weight was put into the reddest HB track, which minimizes the number of PAGB stars. The resulting fit is still a bimodal mass distribution, but this is only because we are excluding those intermediate-mass tracks that lead to PEAGB behavior, given that they produce far too many UVbright stars. The resulting fit, given in Figure 13, shows only slightly better agreement with the data. When there are fundamental discrepancies between the models and data, it is not very meaningful to quantify the goodness of fit, but we do so here for completeness. Our fit minimized a Maximum Likelihood statistic instead of $\chi^{2}$, so we provide an effective $\chi^{2}$ $\left(\chi_{\mathrm{eff}}^{2}\right.$; Dolphin 2002) for readers more familiar with such fitting: $\chi_{\text {eff }}^{2}=1.6$. The fit is strongly ruled out at $38 \sigma$.

Using other PAGB models in the literature, such as those of VW94, does not produce better agreement. In Figure 14, we take the bimodal mass distribution of Figure 11 to populate the EHB, but evolve the red HB stars along one of the VW94 PAGB tracks instead of our own. In Figure 14, the left-hand panels show the H-burning tracks of VW94, while the righthand panels show their He-burning tracks. CMD simulations are shown for main sequence masses of $1,1.5$, and $2 M_{\odot}$ for both types of PAGB tracks. The corresponding luminosity functions are shown in Figure 15. The same PAGB problem persists: for low-mass PAGB tracks, too many UV-bright stars appear at the bright end of our observed range, while for highmass PAGB tracks, the UV-bright stars extend to magnitudes much brighter than observed. This result should not be surprising, given the agreement between our PAGB tracks and those of VW94 in Figures 5 and 8. Thus for all the models in hand, the discrepancies between the predicted and observed distributions of PAGB stars seem unavoidable.

\subsection{Luminosity Gap}

We now turn our attention to the luminosity gap between the EHB and post-HB phases. This gap is very pronounced in the simulations so far, in contrast to the observations. There are several parameters that can affect the distribution of $\mathrm{HB}$ and post-HB stars and consequently the size of the predicted luminosity gap, including metallicity, helium abundance, and extinction. We will explore each of these in turn.

First we consider metallicity. In Figure 16, we show simulations assuming the same bimodal distribution of mass given in Figure 11, but for $[\mathrm{Fe} / \mathrm{H}]$ values of -0.25 and -0.45 instead of solar. The corresponding luminosity functions are shown in Figure 17 . We next combine $[\mathrm{Fe} / \mathrm{H}]$ values of 0 , -0.25 , and -0.45 with relative weights of $25 \%, 50 \%$, and $25 \%$, respectively, to approximate the metallicity distribution of Worthey et al. (2004). The resulting simulation (given in the lower left-hand panel of Figure 16) shows some blurring of the luminosity gap, but not enough to account for the observations. Thus, neither a combination of metallicities nor a single metallicity will account for the absence of a luminosity gap.

Next, we explore the effects of enhancing the helium abundance. With all other parameters (age, metallicity, mass loss, 

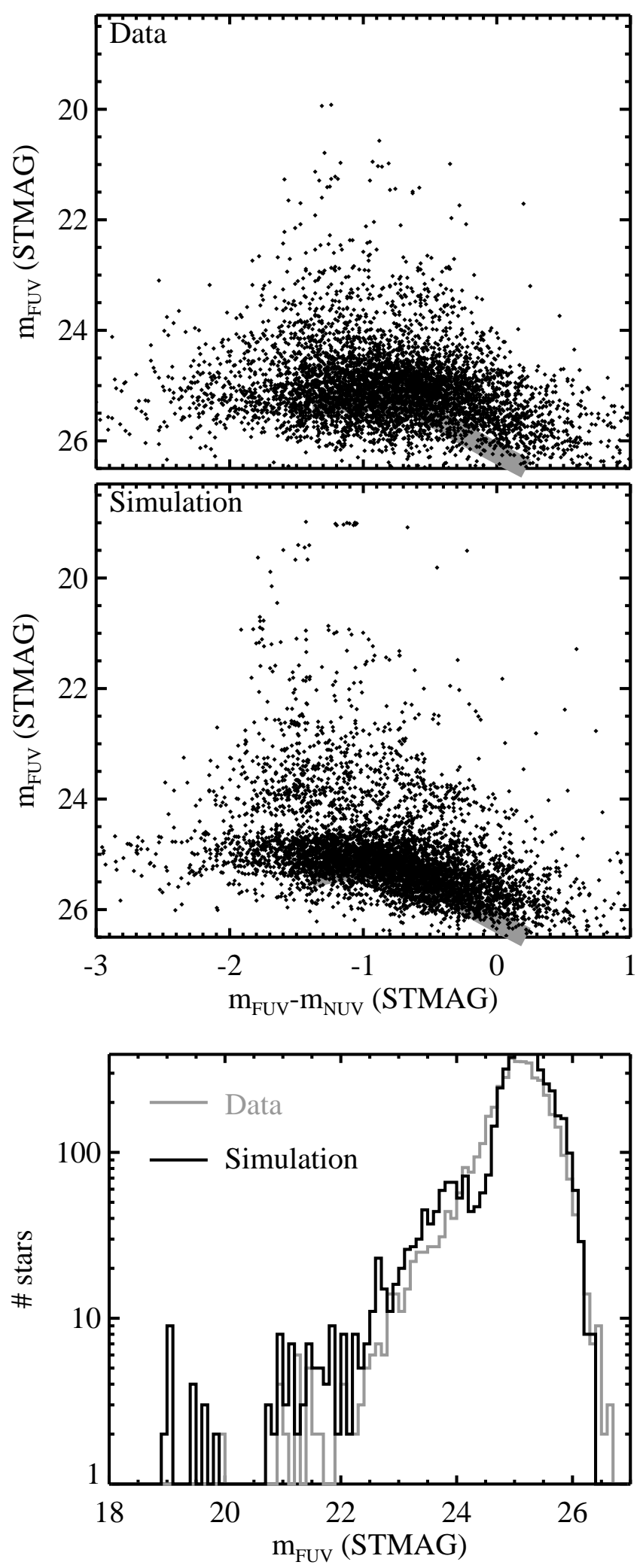

FIG. 13.- Top: The observed far-UV CMD of M32. Middle: A simulated CMD arising from a bimodal distribution of mass on the $\mathrm{HB}$, where the weights of the EHB tracks are determined by a Maximum Likelihood statistic, and the remainder of the population falls on the reddest HB track. Bottom: The comparison of the observed and simulated luminosity functions for those stars at $-2 \leq\left(m_{F U V}-m_{N U V}\right) \leq 0 \mathrm{mag}$. etc.) held fixed, a population will form more EHB stars as the helium abundance is increased (Greggio \& Renzini 1990). This is because an enhanced helium abundance reduces the mass at the main sequence turnoff, thus leading to HB stars of lower mass, and because helium-rich EHB stars of a given envelope mass have a higher effective temperature (see Figure 10). High values of $\Delta Y / \Delta Z$ are thus one of the possible explanations for producing EHB stars in relatively metalrich elliptical galaxies (e.g., O'Connell et al. 1999; YDK97; DRO93). In Figure 16, we show a simulation with the same bimodal mass distribution employed in the previous simulations, but with $Y$ values of 0.28 and 0.43 for the EHB stars, corresponding to $\Delta Y / \Delta Z=4$ and 19 , respectively. The corresponding luminosity functions are shown in Figure 17. Increasing the helium abundance has two notable effects on the UV CMD: it increases the size of the luminosity gap between the EHB and AGBM phases, and it increases the size of the AGBM population relative to the EHB. Both of these effects serve only to increase the discrepancy between the models and the observed UV CMD. This $Y$ sensitivity of the UV CMD was one of the main motivations for our observations, given the difficulty in measuring $Y$ in elliptical galaxies, but our UV CMD is apparently inconsistent with an enhanced $Y$.

Finally, we explore the effects of extinction. Because the effects of extinction are so much stronger in the UV than in the optical, a relatively small dispersion in $E(B-V)$ can produce a large spread in the UV. As an example, we assume a Gaussian distribution of $E(B-V)$ values, with a mean value of 0.08 mag (Schlegel et al. 1998), a one-sigma width of $0.04 \mathrm{mag}$, and the mean Galactic extinction law (Fitzpatrick 1999); the Gaussian was truncated to avoid negative (unphysical) values of $E(B-V)$. The resulting CMD is shown in Figure 16, and the luminosity function is shown in Figure 17. This small dispersion in extinction does produce much better agreement with the observations. In particular, the luminosity gap in the simulation is much less pronounced.

Given the promising results of the simulation with an extinction spread, we decided to improve this simulation by replacing the arbitrary bimodal mass distribution used in Figure 11 with a fit to the observed CMD, based on the minimization of a Maximum Likelihood statistic, as in Figure 13. Again, we enforced the constraint that the total number of stars on all tracks must be consistent with the SEF of $3 \times 10^{-3}$ stars $_{\mathrm{yr}^{-1}}$. The weights for the individual EHB tracks were allowed to float freely within this constraint, and any remaining weight was put into the reddest HB. All other parameters were held fixed: $[\mathrm{Fe} / \mathrm{H}]=-0.25$ (the peak in the M32 metallicity distribution; Worthey et al. 2004), solar $Y$, and a Gaussian distribution of $E(B-V)$ with a mean of 0.08 mag and a one-sigma width of 0.04 mag. The result is shown in Figure 18, which compares the CMD and luminosity function in the data and best fit. Although this fit is much better than our earlier attempts $\left(\chi_{\text {eff }}^{2}=1.2\right)$, there are still clear discrepancies, and the fit is formally ruled out at $15 \sigma$. In the simulation, the UVbright post-HB population includes too many stars that extend to brighter magnitudes than observed, but as discussed previously, there is no way to address this problem with the models at our disposal. Furthermore, the EHB is more sloped in the simulation than in the observed CMD; this may be due to the particular distribution of age, $[\mathrm{Fe} / \mathrm{H}], Y$, and mass in the observed population (including correlations between these parameters), but given the number of free parameters and the limitations of these models, we will not try to resolve this discrepancy here. 

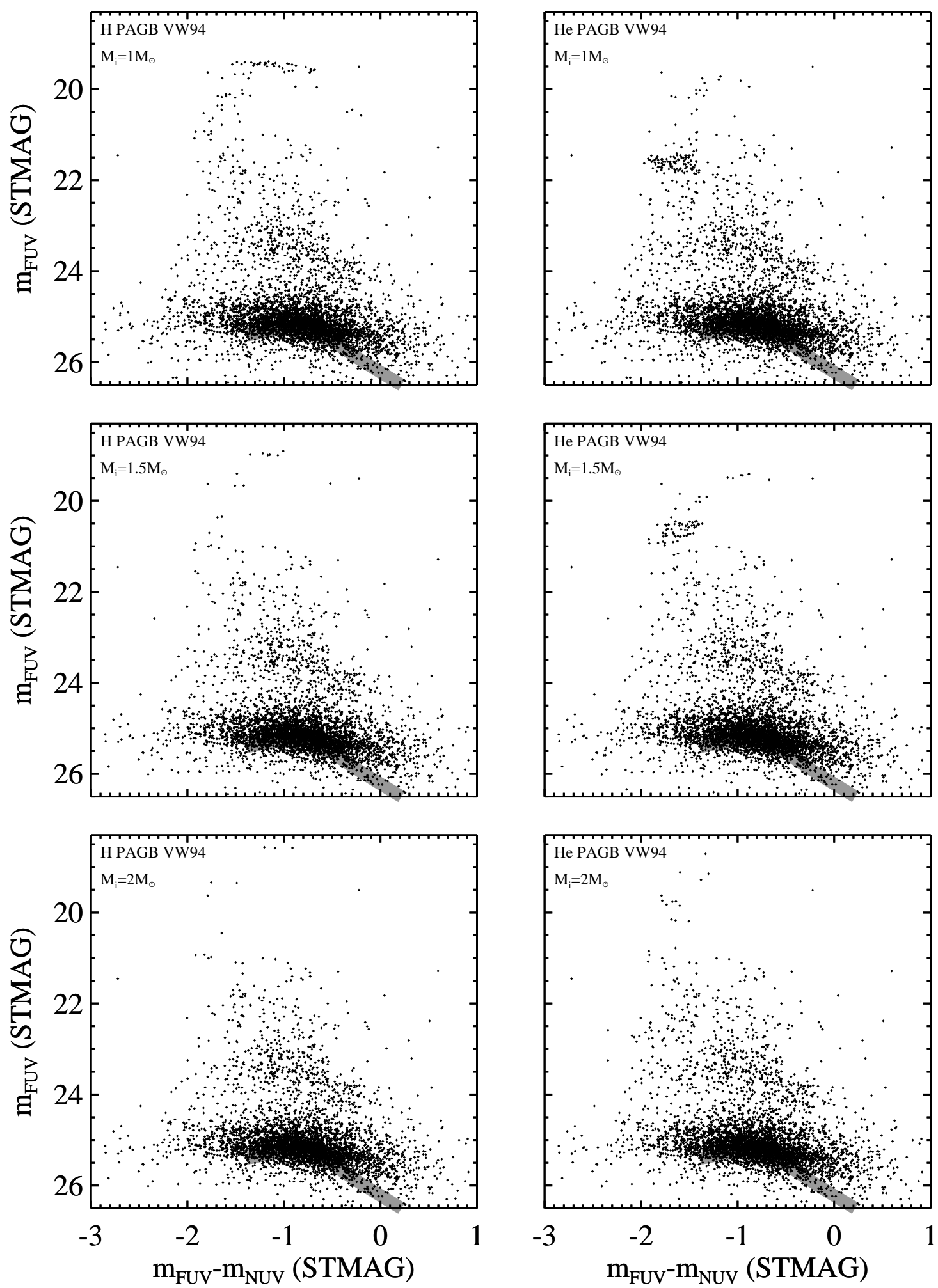

FIG. 14. - Simulated CMDs corresponding to a bimodal distribution of mass on the HB, as given in Figure 11, but with the red HB stars evolving along the PAGB tracks of VW94 instead of our own. Each panel is labeled with the main sequence mass and the PAGB class. 

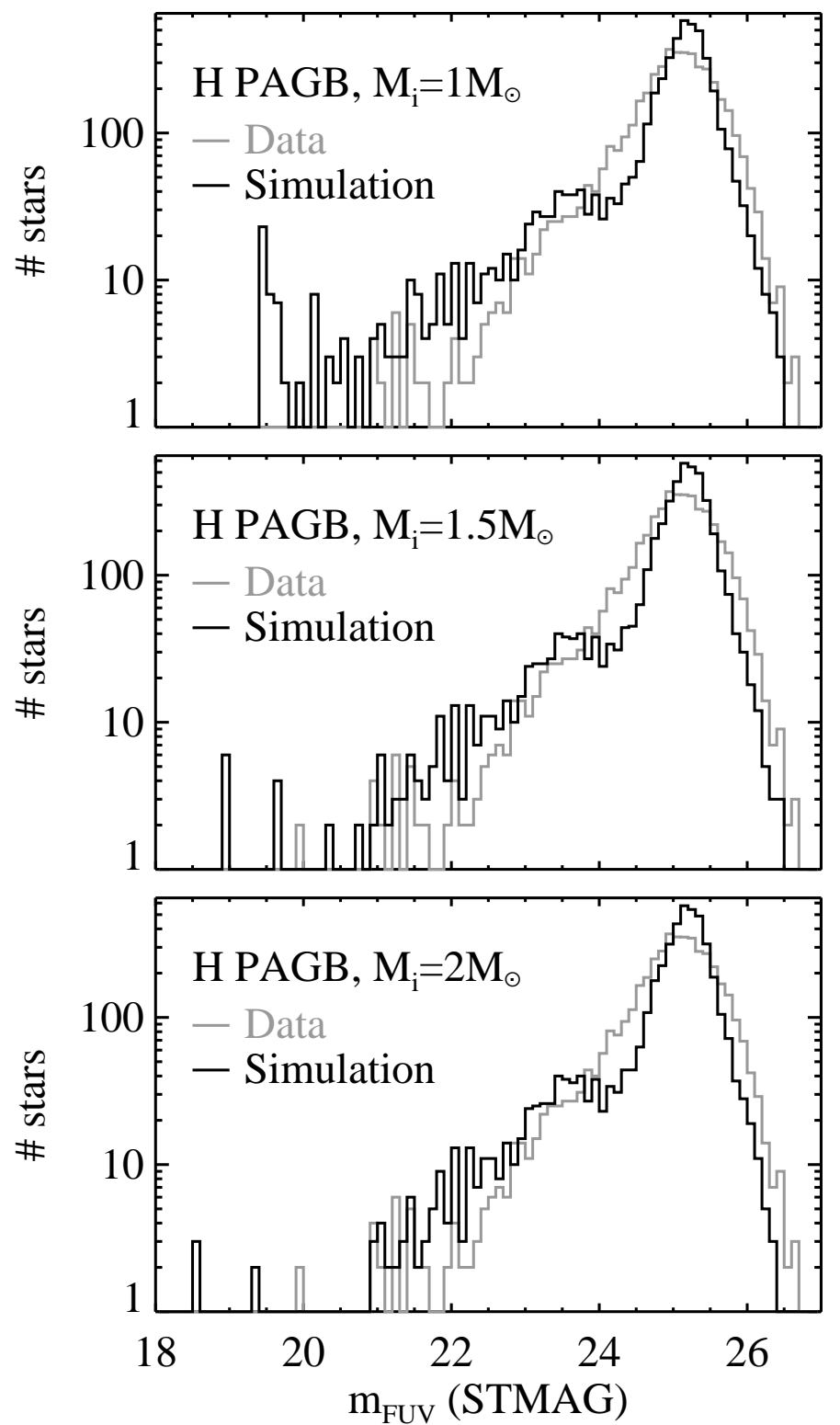
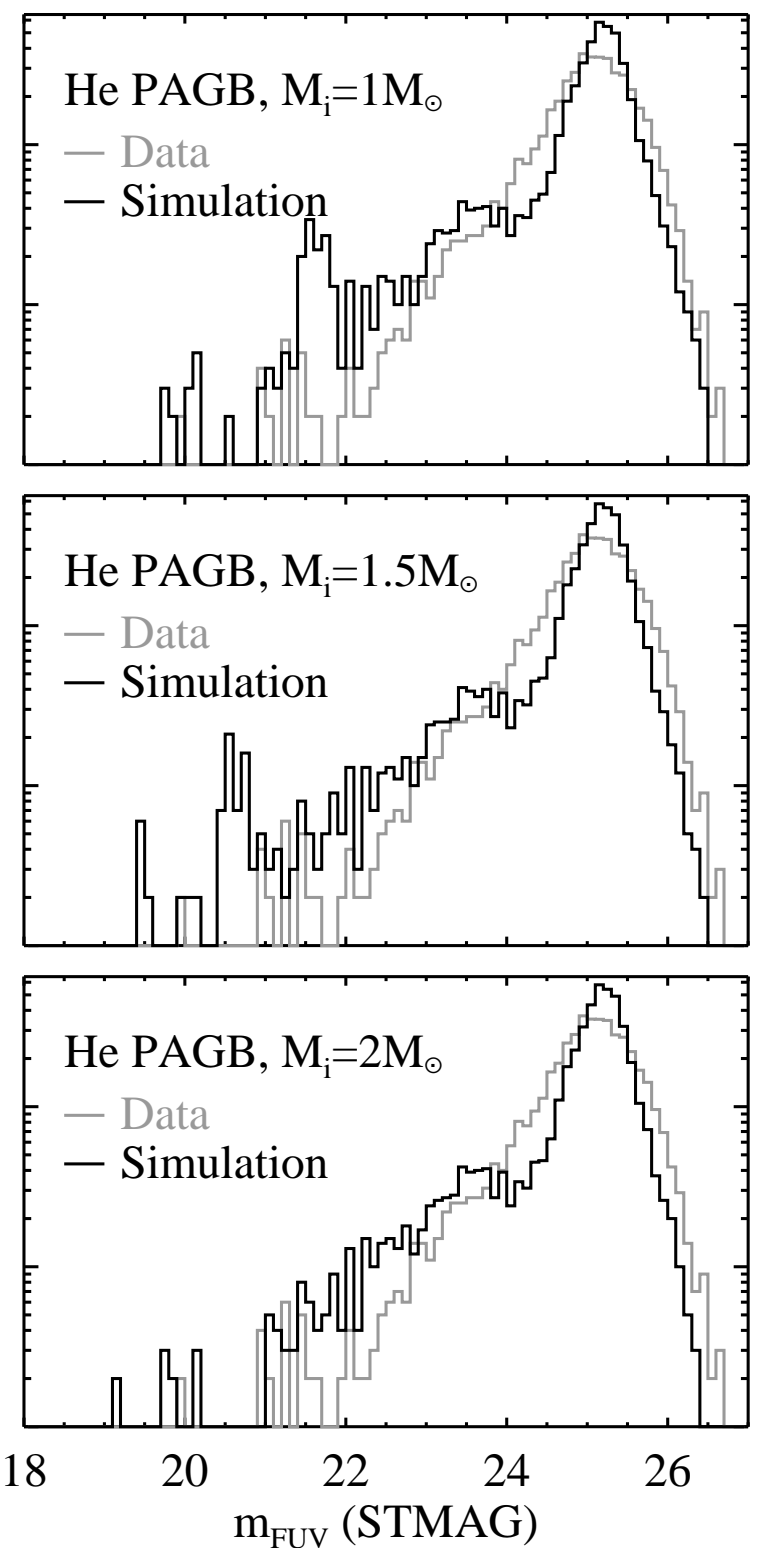

FIG. 15. - Luminosity functions for each of the simulated CMDs from Figure 14 (black histograms) compared to the observed far-UV luminosity function (grey histograms). Depending upon the assumed mass of the PAGB stars, the simulations produce PAGB stars far brighter than observed, and/or too many PAGB stars near the bright end of our observed range of luminosities.

\section{DISCUSSION}

We have presented a UV CMD for a field in the center of the compact elliptical galaxy M32. As found in our previous analysis of the near-UV data alone, we find that the UV light in M32 is clearly due to a minority $(\sim 2 \%)$ population of EHB stars, and that there is a dearth of UV-bright post-HB stars (AGBM, PEAGB, and PAGB stars) when comparing the observed CMD to the expectations of stellar evolution models. Although the presence of EHB stars in metal-rich elliptical galaxies has often been attributed to the possible enhancement of helium at high metallicity (see O'Connell 1999 and references therein), our UV CMD is inconsistent with a supersolar $Y$ in the EHB population. Compared to the models, we find a wider far-UV luminosity spread in the EHB, and little (if any) luminosity gap between the EHB and AGBM phases.

The inconsistency between the models and data can be largely rectified by assuming a small dispersion $(\sigma \approx$
$0.04 \mathrm{mag})$ in $E(B-V)$ around the mean value of $0.08 \mathrm{mag}$. This dispersion in extinction gives our simulated EHB a luminosity width nearly identical to that observed, but the agreement with the observed CMD is still not perfect, due to a stronger tilt in the simulated EHB, and to the larger predicted number of UV-bright stars (see previous section).

If we are really detecting a dispersion in extinction along the sightlines to M32's evolved stars, it is not clear where this extinction lies. We see no evidence of large-scale extinction patchiness in our field (comparing the CMD of distinct regions shows no shift in mean EHB luminosity or EHB width). A variation in foreground Galactic extinction seems improbable, given the fraction of the Galaxy intercepted by the small STIS field. The M31 disk would be a better candidate for variable extinction, but it is unclear if M32 lies behind the M31 disk or in front of it (Worthey et al. 2004; Ford et al. 1978; Paulin-Henriksson 2002). The variable extinction could be due to a small amount of dust in the center of M32, but 

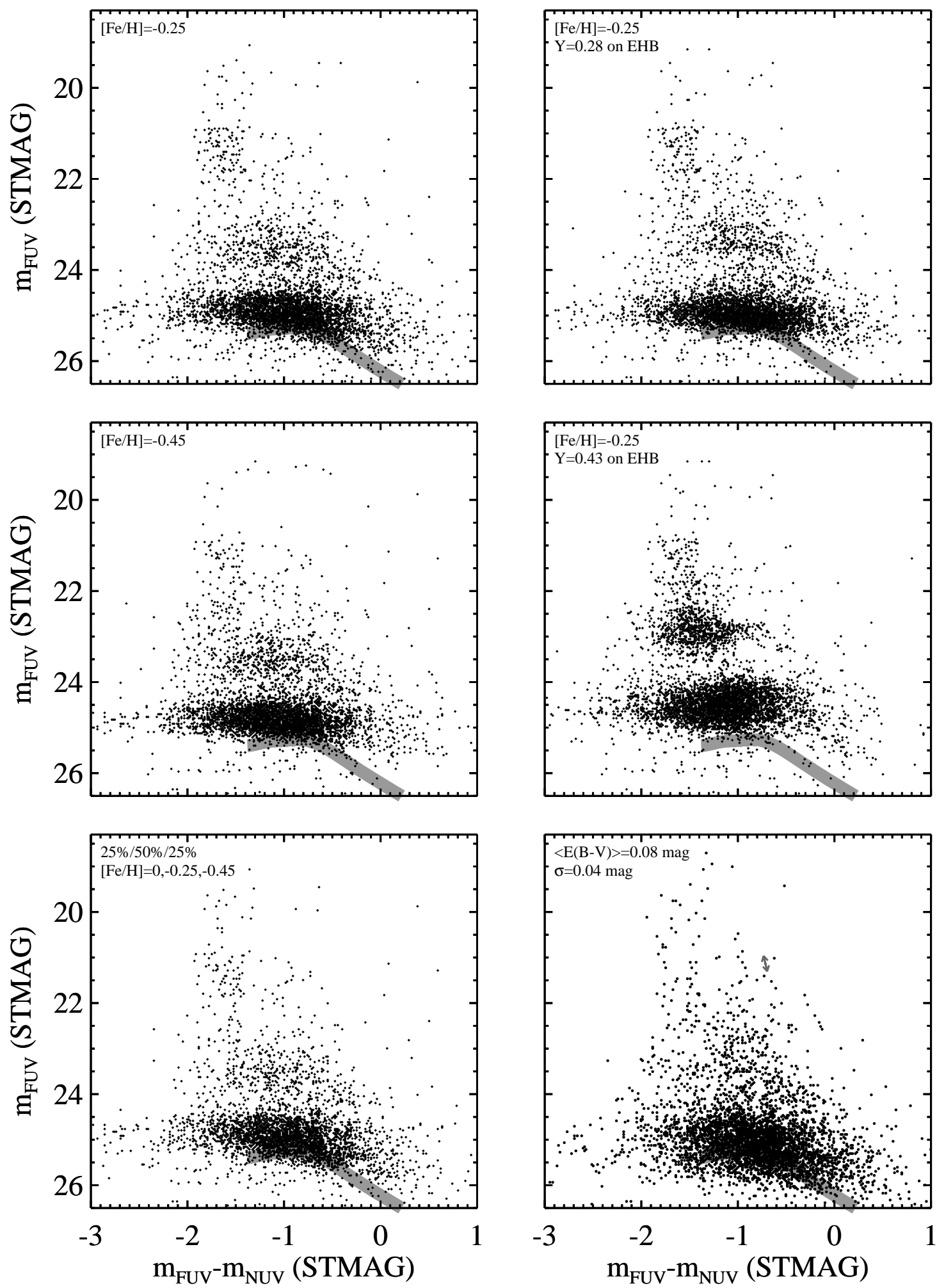

FIG. 16.- Simulated CMDs corresponding to a bimodal distribution of mass on the HB, as in Figure 11, but with variations in $[\mathrm{Fe} / \mathrm{H}], Y$, and extinction. A distribution of $[\mathrm{Fe} / \mathrm{H}]$ (lower left-hand panel) does not blur the luminosity gap enough to match the observed CMD, but a Gaussian distribution of $E(B-V)$ (lower right-hand panel) does. The extinction vector is shown in grey in the lower left-hand panel, scaled to the CMD displacement arising from $E(B-V)=0.08 \pm$ $0.02 \mathrm{mag}$. Enhancing the helium abundance for the EHB population causes a more prominent luminosity gap, which is very discrepant with the observations. 

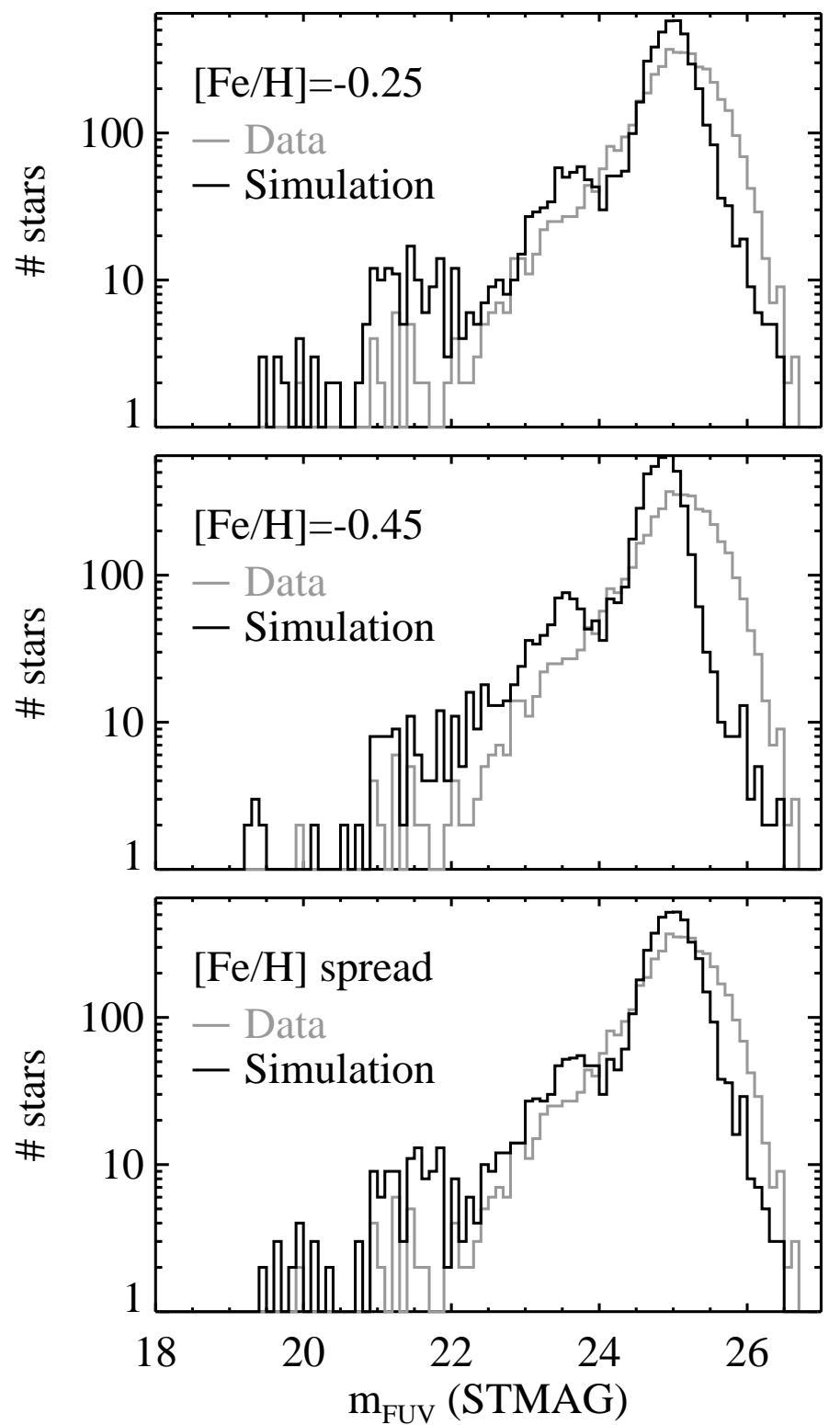
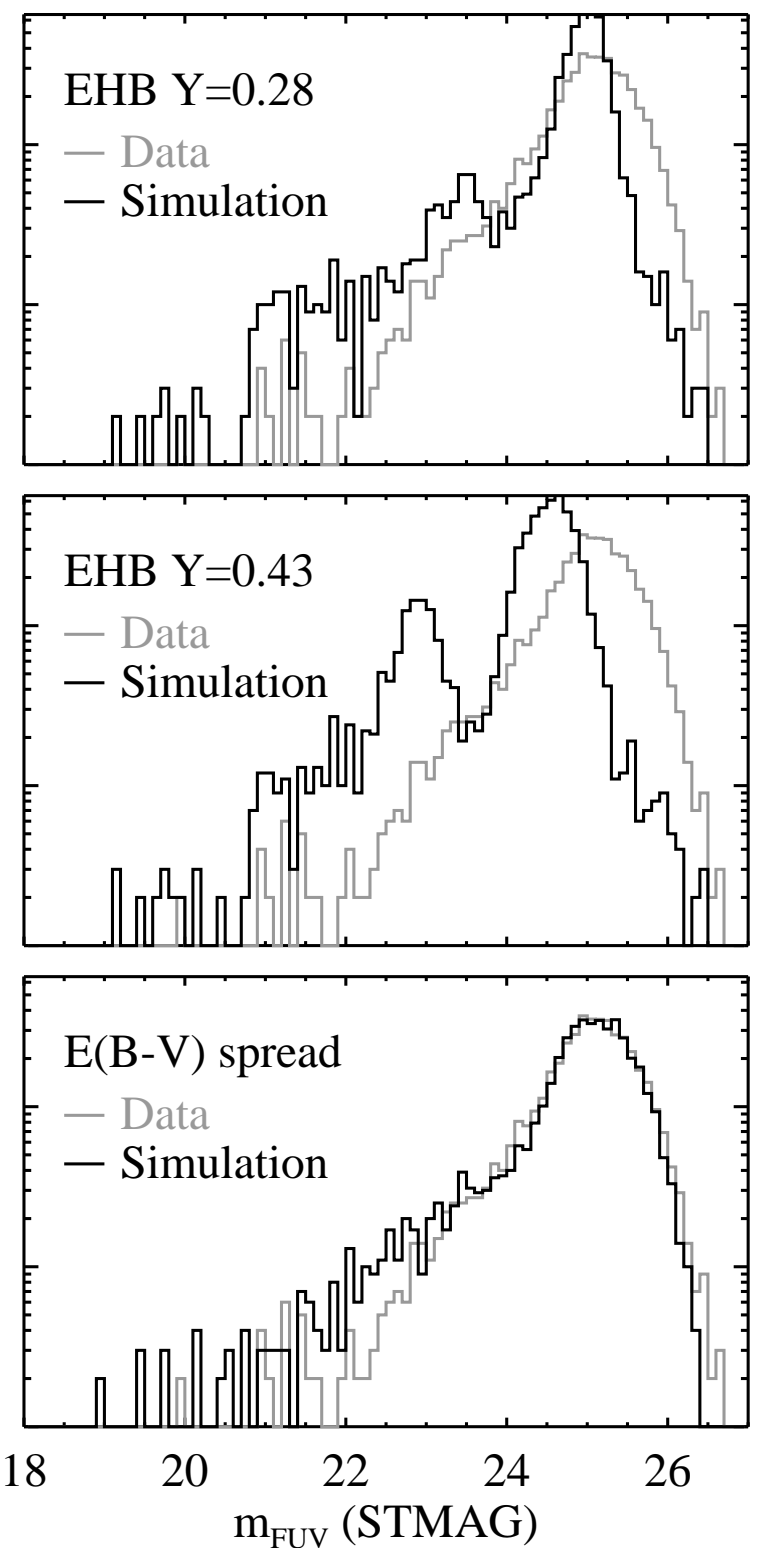

FIG. 17. - Luminosity functions for each of the simulated CMDs from Figure 16 (black histograms) compared to the observed far-UV luminosity function (grey histograms).

observations suggest there is not much (Corbin et al. 2001). The varying amount of dust could conceivably be localized to these evolved stars, given the mass lost in their earlier evolutionary phases. Our far-UV bandpass falls to the blue of the $220 \mathrm{~nm}$ extinction bump, while our near-UV bandpass falls on top of this bump, so appropriately deep observations near the $U$-band would be useful in explorations of this extinction possibility.

As discussed by Brown et al. (2000b), there are several possible explanations for the lack of PAGB stars in our field. The majority of the PAGB stars might be crossing the HR diagram on a thermal timescale instead of a nuclear timescale; this could happen if the final mass ejection on the AGB is triggered by a helium-shell flash that leaves the star out of thermal equilibrium (Käufl et al 1993; Greggio \& Renzini 1999). PAGB stars will also cross the HR diagram more rapidly if they are more massive, but this is problematic, because such stars will cross at luminosities much brighter than we observe; such luminosities would also imply an AGB tip luminosity much brighter than observed (Greggio \& Renzini 1999). Another possibility is that the PAGB stars are obscured by circumstellar material, but this solution is also problematic, given the rapid thinning times for material surrounding post-AGB stars (Käufl et al. 1993).

Compared to other elliptical galaxies, M32 has an extremely weak UV upturn (Burstein et al. 1988). In principle, all of its UV emission could arise from the PAGB descendents of an entirely red HB population. Instead, we find that nearly all of its UV emission is due to a minority population of EHB stars and their AGBM progeny. Most of the HB stars reside on the red end of the $\mathrm{HB}$, but only a small fraction of their expected PAGB descendents are present. These findings have significant implications for studies trying to use the UV upturn as a tracer of age in old populations. The UV upturn has great potential as a tracer of age in a population, because as a population ages, the mass at the main sequence turnoff 
decreases, leading to HB stars of lower mass and hotter temperatures (if RGB mass loss is held fixed). For this reason, several studies have tried to map the strength of the UV upturn in elliptical galaxies as a function of redshift (Brown et al. 1998; Brown et al. 2000a; Brown et al. 2003; Lee et al. 2005; Ree et al. 2007). Unfortunately, at the present time, the many parameters that govern the presence of EHB stars in a population are poorly constrained; one can tune the assumptions (chemical evolution, RGB mass loss, binaries, etc.) so that the sudden onset of the UV upturn in a population can occur at nearly any age greater than a few Gyr. Some models (e.g., those in Ree et al. 2007) assume that EHB stars fade rapidly beyond $z \sim 0.1$, such that the UV emission from populations at $z \gtrsim 0.3$ should be completely dominated by PAGB stars. The results we have presented here show that this is not necessarily the case. We do not know the age distribution in M32, but at least part of the population is old enough to host EHB stars. M32 is an elliptical galaxy at $z=0$ with far fewer PAGB stars than one would expect. If we observed M32 in the past, it would have even fewer PAGB stars, because the PAGB stars would be more massive and evolve more rapidly. The population of M32 implies that PAGB stars are not a significant source of UV emission, and that at any redshift where significant UV emission is present in a population, it is likely due to either EHB stars or residual young populations; this is true even for redshifts at the high end of the range explored in UV upturn studies ( $z=0.55$; Brown et al. 2000a). Models that predict a rapid fading of UV emission with increasing redshift should likely be revised, such that the UV emission fades even more dramatically, because the PAGB contribution to such emission is likely overestimated.

Support for proposal 9053 is provided by NASA through a grant from STScI, which is operated by AURA, Inc., under NASA contract NAS 5-26555. We are grateful to P. Stetson for his DAOPHOT code, and to A. Renzini for interesting discussions.

\section{REFERENCES}

Brown, T.M., Bowers, C.W., Kimble, R.A., \& Ferguson, H.C. 2000a, ApJ, 529, L89

Brown, T.M., Bowers, C.W., Kimble, R.A., Sweigart, A.V., \& Ferguson, H.C. 2000b, ApJ, 532, 308

Brown, T.M., Ferguson, H.C., Davidsen, A.F., \& Dorman, B. 1997, ApJ, 482, 685

Brown, T.M., Ferguson, H.C., Deharveng, J.-M., \& Jedrzejewski, R.I. 1998 , ApJ, 508, L139

Brown, T.M., Ferguson, H.C., Smith, E., Bowers, C.W., Kimble, R.A., Renzini, A., \& Rich, R.M. 2003, ApJ, 584, L69

Brown, T.M., Kimble, R.A., Ferguson, H.C., Gardner, J.P., Collins, N.R., \& Hill, R.S. 2000c, AJ, 120, 1153

Burstein, D., Bertola, F., Buson, L.M., Faber, S.M., \& Lauer, T.R. 1988, AJ, 95, 1298

Cassisi, S., Castellani, V., Degl'Innocenti, S., Piotto, G., \& Salaris, M. 2001, A\&A, 366, 578

Castelli, F., \& Kurucz, R.L. 2003, in IAU Symposium 210, Modeling of Stellar Atmospheres, eds. N. Piskunov, W.W. Weiss, \& D.F. Gray, poster A20, astro-ph/0405087

Corbin, M.R., O’Neil, E., \& Rieke, M.J. 2001, AJ, 121, 2549

Dolphin, A.E. 2002, MNRAS, 332, 91

Dorman, B., O’Connell, R.W., \& Rood, R.T. 1995, ApJ, 442, 105

Dorman, B., Rood, R.T., \& O’Connell, R.W. 1993, ApJ, 419, 596 (DRO93)

Ferguson, H.C., et al. 1991, ApJ, 382, L69

Ferraro, F.R., Messineo, M., Fusi Pecci, F., De Palo, M.A., Straniero, O., Chieffi, A., \& Limongi, M. 1999, AJ, 118, 1738

Fitzpatrick, E.L. 1999, PASP, 111, 63

Ford, H.C., Jacoby, G.H., \& Jenner, D.C. 1978, ApJ, 223, 94

Freedman, W.L., \& Madore, B.F. 1990, ApJ, 365, 186

Fruchter, A.S., \& Hook, R.N. 2002, PASP, 114, 144

Greggio, L., \& Renzini, A. 1990, ApJ, 364, 35

Greggio, L., \& Renzini, A. 1999, in UV Astronomy in Italy, ed. L.M. Buson \& Domitilla De Martino (Fierenze: Soc. Astron. Italiana), 691
Hesser, J.E., Harris, W.E., VandenBerg, D.A., Allwright, J.W.B., Shott, P., \& Stetson, P.B. 1987, PASP, 99, 739

Käufl, H.U., Renzini, A., \& Stanghellini, L. 1993, ApJ, 410, 251

Landsman, W., Moehler, S., Napiwotzki, R., Heber, U., Sweigart, A., Catelan, M., \& Stecher, T. 2000, in The Galactic Halo: From Globular Cluster to Field Stars, Proceedings of the 35th Liege International Astrophysics Colloquium, ed. A. Noels, P. Magain, D. Caro, E. Jehin, G. Parmentier, \& A.A. Thoul (Liege: Institut d'Astrophysique et de Geophysique), 515

Lee, Y.-W., et al. 2005, ApJ, 619, L103

Montegriffo, P., Ferraro, F.R., Fusi Pecci, F., \& Origlia, L. 1995, MNRAS, 276,739

O’Connell, R.W. 1999, ARA\&A, 37, 603

Park, J.-H., \& Lee, Y.-W. 1997, ApJ, 476, 28

Paulin-Henriksson, S., et al. 2002, ApJ, 576, L121

Proffitt, C. 2003, ISR STIS 2003-01: Absolute Flux Calibration of STIS MAMA Imaging Modes

Ree, C.H., et al. 2007, ApJS, 173, 607

Reimers, D. 1975, Mém. Royal Soc. Liège, 8, 369

Renzini, A. 1998, AJ, 115, 2459

Robertson, J.W., \& Faulkner, D.J. 1972, 171, 309

Schlegel, D.J., Finkbeiner, D.P., \& Davis, M. 1998, ApJS, 500, 525

Stetson, P. 1987, PASP, 99, 191

Tantalo, R., Chiosi, C., Bressan, A., \& Faggotto, F. 1996, A\&A, 311, 361

Walterbos, R.A.M., \& Kennicutt, R.C. 1987, A\&AS, 69, 311

Woodgate, B.E., et al. 1998, PASP, 110, 1183

Worthey, G. 1994, ApJS, 95, 107

Worthey, G., Mateo, M., Alonso-García, J., \& España, A.L. 2004, PASP, 116, 295

Vassiliadis, E., \& Wood, P.R. 1994, ApJS, 92, 125 (VW94)

Yi, S., Demarque, P., \& Kim, Y.-C. 1997, 482, 677 (YDK97)

Yi, S., Lee, Y.-W., Woo, J.-H., Park, J.-H., Demarque, P., \& Oemler, A., Jr. 1999, ApJ, 513, 128 

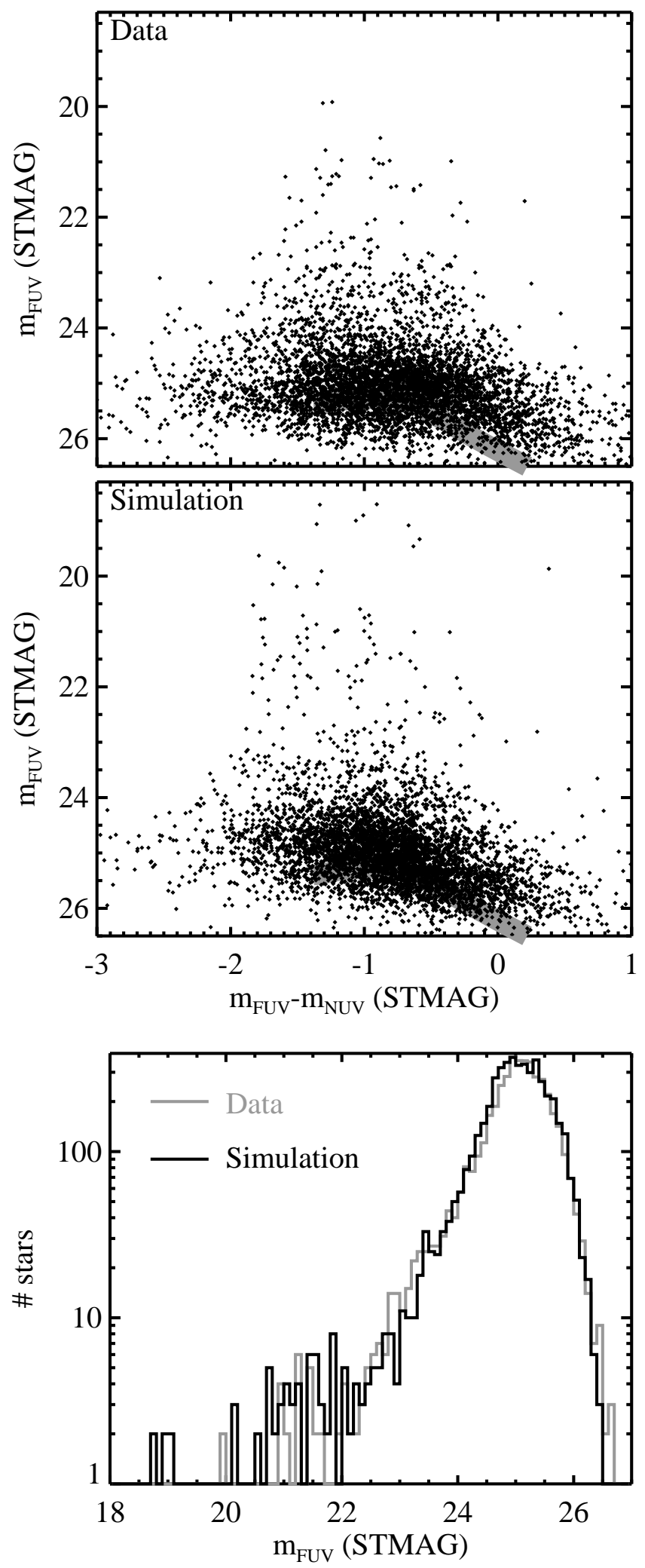

FIG. 18. - Top: The observed far-UV CMD of M32. Middle: A simulated CMD arising from a bimodal distribution of mass on the HB, where the weights of the EHB tracks are determined from a fit using a Maximum Likelihood statistic, and the remainder of the population falls on the reddest HB track. The $[\mathrm{Fe} / \mathrm{H}]$ is fixed at -0.25 , the $Y$ is fixed at the solar value, and there is a Gaussian distribution of $E(B-V)$ with a mean of $0.08 \mathrm{mag}$ and a one-sigma width of 0.04 mag. Bottom: The comparison of the observed and simulated luminosity functions. 